\title{
Evaluation of saponin-rich extracts as natural alternative emulsifiers: A comparative study with pure Quillaja Bark saponin
}

\author{
Tatiana B. Schreiner ${ }^{\text {a, b }}$, Giovana Colucci ${ }^{\text {a }}$, Arantzazu Santamaria-Echart ${ }^{\text {a }}$, Isabel P. Fernandes ${ }^{\text {a }}$, \\ Madalena M. Dias $^{\text {b }}$, Simão P. Pinho ${ }^{\text {a,"*, Maria Filomena Barreiro }}{ }^{\text {a, }}$ \\ ${ }^{a}$ Centro de Investigação de Montanha (CIMO), Instituto Politécnico de Bragança, Campus de Santa Apolónia, 5300-253 Bragança, Portugal \\ ${ }^{\mathrm{b}}$ Laboratory of Separation and Reaction Engineering - Laboratory of Catalysis and Materials (LSRE-LCM), Polytechnic Institute of Bragança, Campus Santa Apolónia, \\ 5301-253 Bragança, Portugal
}

\section{H I G H L I G H T S}

- T. terrestris, T. foenum-graecum, R. aculeatus extracts evaluated as emulsifiers.

- Extract's emulsifier properties compared with pure Quillaja saponin.

- T. Terrestris is the most promising extract with a CMC of $0.59 \mathrm{~g} \mathrm{~L}^{-1}$.

- The extract's saponin content does not always rule their emulsifying properties.

- The extract's foam capacity is 3-fold lower than the one of Quillaja saponin.

\section{A R T I C L E I N F O}

\section{Keywords:}

Saponin

Plant extracts

Emulsifying properties

Tribulus terrestris

Trigonella foenum-graecum

Ruscus aculeatus

\section{G R A P H I C A L A B S T R A C T}

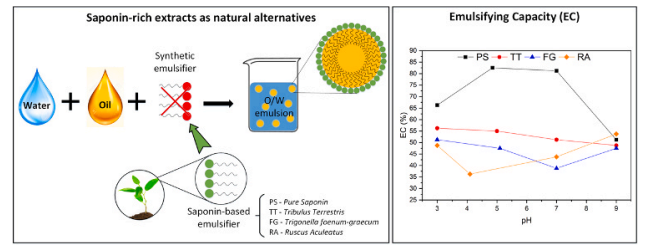

\section{Introduction}

The importance of emulsifiers to form stable emulsions with functional attributes and suitable shelf lives is a relevant topic for several industries. Nowadays, most of the emulsifiers used at industrial level are synthetic, such as Tweens [1] and Spans [2]. These products are

\footnotetext{
* Corresponding authors.

E-mail addresses: spinho@ipb.pt (S.P. Pinho), barreiro@ipb.pt (M.F. Barreiro).
} 
becoming increasingly associated with health and environmental problems encouraging the industrial sector to search for "label-friendly" alternatives [3]. In this way, a strong effort is being made to identify, study, characterise and apply natural emulsifiers [4].

Saponins are molecules with polar sugar moieties attached to nonpolar triterpene or steroid units, making them amphiphilic structures with high surface-activity [5]. Saponins' biological and physicochemical properties led to their use in several traditional and industrial applications (food, cosmetics, beverages, personal care, and pharmaceuticals) [6-8]. Moreover, they can be the basis to develop new strategies leading to the re-evaluation of the current emulsion formulation practices [9].

One of the first reported works exploiting quillaja saponins as alternative emulsifiers was carried out by Pekdemir et al. [10], who successfully performed the emulsification of crude oil at low saponin concentrations $(0.1 \mathrm{wt} \%)$. Thereafter, several studies are being carried out to elucidate the use of quillaja saponins to stabilise oil-in-water emulsions, which were reported to be highly stable to heat treatment $\left(30-90{ }^{\circ} \mathrm{C}\right)$ [11], $\mathrm{pH}$ change (pH 3-8) [12], high ionic strength media (up to $300 \mathrm{mM} \mathrm{NaCl}$ ) [5], and storage time (one month at $5-55^{\circ} \mathrm{C}$ ) [5, 13]. Quillaja saponin's emulsifying properties were also tested with different oils (long and medium-chain triglycerides, e.g. corn oil and miglyol 812) [14]. Works addressing the determination of interfacial properties, foaming characteristics, and critical micelle concentration (CMC) were also conducted, leading to outcomes showing their high surface activity (surface tension of $40.2 \mathrm{mN} / \mathrm{m}$ at $0.5 \mathrm{~g} \mathrm{~L}^{-1}$ ) [15], good foam formation capacity (foaming speed above $1.5 \mathrm{~mm} / \mathrm{s}$ ) [16] and low CMC (below $0.7 \mathrm{~g} \mathrm{~L}^{-1}$ ) [17].

The possibility of using saponin extracts from different botanical sources started also to be addressed, with one of the pioneer works carried out by Weiss and co-workers, who studied yucca saponin from Yucca schidigera (9.5 wt\%) [18], ginseng from Panax ginseng (7 wt\%) [19], and oat bran from Avena sativa L. (4.6 wt\% saponin content) [20] to stabilise emulsions. Generally speaking, the emulsions were stable at the $\mathrm{pH}$ range of $4-9$ and over heat treatments up to $50^{\circ} \mathrm{C}$. Regarding the ionic strength, except for ginseng emulsion $(\leq 100 \mathrm{mM} \mathrm{NaCl})$, all showed high instability with salt addition $\left(\mathrm{NaCl}\right.$ and $\left.\mathrm{CaCl}_{2}\right)$. Moreover, the storage time was also evaluated, with oat bran extract giving rise to 42 days of stability (at 5 and $25^{\circ} \mathrm{C}$ ), contrasting with yucca that showed coalescence after 28 days. Zhu et al. [21] performed a similar study using tea saponin extract from Camellia lutchuensis (51.8 wt\% saponin content). The emulsions showed to be stable to droplet aggregation and phase separation in a $\mathrm{pH}$ range of $\mathrm{pH}$ 3-9 and thermal processing from $30{ }^{\circ} \mathrm{C}$ to $90^{\circ} \mathrm{C}$. Besides, they remained stable along 30 days of storage (at 5,25 , and $55^{\circ} \mathrm{C}$ ). Recently, Bouhoute et al. [22] prepared extracts with a saponin content of 10.4-39.1 wt\% from argan shells, identifying that all of them were capable of producing emulsions with submicron droplets and good physical stability (30 days storage at 5 and $25{ }^{\circ} \mathrm{C}$ ). These studies highlight the potential of using saponin extracts in alternative to saponin pure forms. Moreover, they observed that the saponin content does not always rule the emulsifying properties once the extracts have complex compositions and functionalities capable to interfere in the stability of the produced emulsions.

Face to the increasing interest to use saponin extracts as natural surfactants, the search for new sources become an important topic of study, with several saponin-rich extracts already available commercially. Examples include Puncture vine (Tribulus terrestris), Fenugreek (Trigonella foenum-graecum), and Butcher's broom (Ruscus Aculeatus) matrices. Puncture vine extract finds uses in the treatment of several ailments, exhibiting radical scavenging and antioxidant activity [23], antimicrobial and cytotoxic effects [24]. Fenugreek extract presents antioxidant potential, showing health benefits in the prevention of human diseases involving free radicals [25]. Besides, it is safe for general or specific use according to the Food and Drug Administration (FDA) [26]. Butcher's broom extract, has important pharmacological properties, such as anti-inflammatory activity and action against venous insufficiency [27]. These properties combined with the emulsifying potential of saponins open the possibility to develop functional emulsions, reinforcing the importance to study extracts as emulsifiers.

Having in view the direct use of saponin-rich extracts as emulsifier agents, this work aims to evaluate their potential in the field, and potentiate their application in commercial products as an alternative to the pure saponin form. A complete study was performed for three extracts, namely Puncture vine, Fenugreek, and Butcher's broom extracts, whose performance was compared with pure Quillaja Bark saponin. To de best of our knowledge, there is no report in the literature addressing the study of these plant extracts as emulsifiers. Thus, this is an important study to better understand the performance of saponin-rich extracts from different botanical origins.

\section{Materials and methods}

\subsection{Saponin samples}

In this work, saponin-rich extracts (Tribulus terrestris (TT), Trigonella foenum-graecum (FG), and Ruscus aculeatus (RA)) were acquired from Essência d'um Segredo, a Portuguese company specialised in the commercialisation of natural and natural-derived products. They are hydroethanolic extracts with saponin contents ranging from 20 up to 90 $\mathrm{wt} \%$. TT extract was obtained from the fruits of puncture vine, FG extract was obtained from the fruits of Fenugreek, and RA from the roots of Butcher's broom. They comprise a saponin content of $93.1 \mathrm{wt} \%, 50.3$ $\mathrm{wt} \%$, and $20.3 \mathrm{wt} \%$, respectively. These extracts agree with the levels of residual solvents, heavy metals, aflatoxins, benzopyrenes, and polycyclic aromatic hydrocarbons (PAHs) posed by the pharmacological standards. The microbiological parameters comply with the USP 36-61 standard. Besides, they were absent from allergens, OMG, and BSE/ TSE. All the extracts were acquired in the dry form, having FG and RA extracts a content of $10 \%(\mathrm{w} / \mathrm{w})$ of maltodextrin. Additionally to the studied extracts, a pure saponin (PS) from Quillaja Bark (99.9 wt\%) was used for comparison purposes, being purchased from Panreac. The samples (saponin-rich extracts and pure saponin) were used as received without any further purification.

Based on reported data, the main identified constituents in TT extracts are steroidal saponins (furostanol glycosides) [28,29], together with flavonoids (kaempferol, astragalin, quercetin, kaempferol-3-rhamnoglycoside, rutin, and tribuluside) [30,31], alkaloids [32,33], sterols [34], lignan amides and cinnamic acid amides [35, 36]. Regarding FG extracts, steroidal saponins (furostanols glycosides) [37], alkaloids (trigocoumarin, nicotinic acid, and trigonelline) [38], flavonoids [39], other phenolics (scopoletin, chlorogenic, caffeic, and p-coumaric acids) [40], and salicylate [41] are reported. RA is composed of steroidal [42] and triterpenoid saponins [43] and compounds such as, flavonoids and phenolic acids (schaftosid, orientin, vitexin, rutin, isoquercitrin, nicotiflorin, narcissin, caffeic, and p-coumaric acid) [44], coumarins, sparteine, tyramine and glycolic acid [45].

\subsection{Chemicals}

The solvents used in the solubility tests (ethanol, n-hexane, and ethyl acetate) were of analytical grade and acquired from typical suppliers. For the $\mathrm{pH}$ adjustment, the used $\mathrm{HCl} 0.1 \mathrm{M}$ and $\mathrm{NaOH} 0.1 \mathrm{M}$ standard solutions were also purchased from typical suppliers. The oil used to perform emulsion tests was a refined cosmetic grade sweet almond oil (SAO) (LabChem). The used water was deionised water. All the compounds were used as received without any further purification.

\subsection{Solubility tests}

The solubility tests were performed in order to understand the amphiphilic character of saponin and the selected extracts. The chosen solvents, water, ethanol, ethyl acetate, and n-hexane, selected to cover different polarities, were chosen based on the Arizona scale [46], which 
is quite used for solvent screening in applications related to the extractions and purification of valuable chemicals from biomass matrices. The effect of initial saponin concentration on solubility was firstly investigated using pure saponin from Quillaja Bark as the model system. The initial concentration has varied from 25 to $100 \mathrm{~g}$ of saponin per $1 \mathrm{~kg}$ of water, putting into evidence a proportional growth on the solubility. Thus, the consistency of the experimental procedure was guaranteed. The selected initial concentration for all measurements was fixed in 100 $\mathrm{g}$ of solute per $\mathrm{kg}$ of solvent, due to the high solubility of pure saponin in water. The experiments were carried out using the analytical isothermal shake-flask method at $30{ }^{\circ} \mathrm{C}$, following the general methodology described thoroughly elsewhere $[47,48]$. Briefly, the mixtures were prepared at a scale of $60 \mathrm{~g}$ using a concentration of $100 \mathrm{~g}$ of extract (or pure saponin) per $1 \mathrm{~kg}$ of solvent. The solution was continuously stirred for $24 \mathrm{~h}$ to reach equilibrium, then allowed to rest for $12 \mathrm{~h}$. The amount of soluble and insoluble fractions was determined gravimetrically. For the soluble fraction, a sample $\left(5 \mathrm{~cm}^{3}\right)$ of the saturated liquid phase was collected with a plastic syringe, placed into pre-weighed glass vessels, and weighed immediately. Next, the solvent was left to evaporate at ambient temperature, followed by final drying in an oven at $50{ }^{\circ} \mathrm{C}$ to avoid sample degradation. After, these samples were left to cool at ambient temperature in a dehydrator with silica gel, during one hour before weighting. This last step was regularly repeated until a constant weight was achieved $( \pm 0.001 \mathrm{~g})$. The measurements were carried out in triplicate and solubility results expressed in $\mathrm{g} / \mathrm{kg}$ solvent.

\subsection{Fourier-transform infrared spectroscopy (FTIR) analysis}

Infrared spectra were acquired using a Fourier-transform infrared (FTIR) spectrophotometer, model MB3000 from ABB Inc, (Quebec, Canada), operating in ATR mode. Spectra were recorded between 650 and $4000 \mathrm{~cm}^{-1}$ at a resolution of $16 \mathrm{~cm}^{-1}$ and cumulative 32 scans. Spectra were acquired using the software Horizon MB v.3.4. FTIR was used to characterise the original samples and the soluble fractions obtained in the solubility studies.

\subsection{Critical micelle concentration assays}

The critical micelle concentration (CMC) was determined by the dye solubilisation technique using a hydrophobic dye (1-[(E)-\{4-[(E)-Phenyldiazenyl] phenyl\} diazenyl]-2-naphthol, known as Sudan III) following the method proposed by Obasi et al. [49]. The measurements were done using an UV-Vis spectrophotometer (Model T70, PG Instruments) at $498 \mathrm{~nm}$ (maximum absorbance of Sudan III). Briefly for the CMC determination, $200 \mu \mathrm{L}$ of the dye solution in $n$-hexane were added to $5 \mathrm{~mL}$ of the saponin or extract aqueous solutions (concentration range $0.025-2.5 \mathrm{~g} / \mathrm{L}$ ). The mixtures enclosed in closed vials were vigorously shaken for $90 \mathrm{~s}$ and allowed to stand (upside down) for 30 min. Then, aliquots of $3 \mathrm{~mL}$ were taken from the bottom phase, transferred into a cuvette, and the absorbance measured. At concentrations below $\mathrm{CMC}$, the dye remains insoluble in the aqueous phase giving rise to zero absorbance. As the micelles start to form, the dye solubilizes in the aqueous phase, the absorbance starts to increase, and a sharp rise in the absorbance is noticed at the CMC point. The experiments were performed at room temperature.

\subsection{Emulsifying capacity and emulsion stability}

The emulsifying capacity (EC), also called emulsifying activity, defines the emulsifier's ability to form an emulsion. The emulsion stability (ES) refers to the ability of an emulsion to resist change in its properties over time. For EC determination, an emulsion was prepared from SAO and water $(\mathrm{W})$, using a SAO/W ratio of 50/50 (w/w), and $3.5 \mathrm{wt} \%$ of the emulsifier following a reported procedure [50]. Briefly, the emulsion was prepared by mixing $1.4 \mathrm{~g}$ of the extract with $20 \mathrm{~g}$ of water followed by the dropwise addition of the oil $(20 \mathrm{~g})$ under continuous stirring at $2000 \mathrm{rpm}$ for $3 \mathrm{~min}$ using a mechanical stirrer (EUROSTAR 40 digital-IKA). After that, $10 \mathrm{~mL}$ of this emulsion were transferred to a falcon tube of $15 \mathrm{~mL}$ and centrifugated at $3200 \mathrm{rpm}$ for 5 min using a centrifuge (5810R - Eppendorf). The total height of the emulsion and the formed emulsified layer's height were measured, and EC calculated according to the Eq. (1). The procedure to determine ES was similar to the one described for EC, with an additional step where the emulsion was subjected to $80^{\circ} \mathrm{C}$ for $30 \mathrm{~min}$ before the centrifugation stage. After cooling, the emulsified layer's height was measured, and Eq. (1) was used to calculate ES.

EC $\quad($ orES $)=\frac{\text { Height of the emulsified layer } \quad(\mathrm{cm})}{\text { Total height } \quad(\mathrm{cm})} 100$

Both EC and ES measurements were carried out without adjusting the $\mathrm{pH}$ (i.e., by using the natural $\mathrm{pH}$ of the emulsion), and by adjusting the $\mathrm{pH}(3,7$, and 9). A $0.1 \mathrm{M} \mathrm{HCl}$ or a $0.1 \mathrm{M} \mathrm{NaOH}$ solution was used, as needed, to adjust the $\mathrm{pH}$.

The morphology of the formed emulsions was evaluated by optical microscopy, being the images acquired with an optical microscope (Nikon Eclipse 50i) attached to a camera (Nikon Digital Sight). The Software NIS-Elements BR was used for the image analysis.

\subsection{Foaming properties}

Foaming properties were evaluated through two parameters: the foaming capacity (FC) and the foaming stability (FS). The used procedure followed the work of Lupatini et al. [51] with minor modifications. Concisely, an aqueous solution of the extract or pure saponin with a content of $2 \mathrm{wt} \%$, was prepared. Then, $10 \mathrm{~mL}$ of each solution was transferred to Falcon tubes of $50 \mathrm{~mL}$, and then vortexed (V-1 plus Bio$\mathrm{san}$ ) for $10 \mathrm{~min}$. The initial height of the sample (before vortexed) and the total height of the foamed sample (after vortexed) were measured, and Eq. (2) used to calculate the FC.

For FS, the foamed sample obtained after vortexed was thermostated at $30{ }^{\circ} \mathrm{C}$ for $1 \mathrm{~h}$, followed by the determination of the total height of the foamed sample. FS was calculated according to Eq. (2).

$F C($ orFS $)=\frac{\text { Total height of the foamed sample }(\mathrm{cm})}{\text { Initial height }(\mathrm{cm})} 100$

Both FC and FS measurements were carried out without adjusting the $\mathrm{pH}$ and using three fixed $\mathrm{pH}$ values (3, 7, and 9).

Two-way analysis of variance (ANOVA) was applied at a significance level of $5 \%$ to evaluate the existence of statistically significant effects of $\mathrm{pH}$ and saponin type, on the foam formation and stability.

\subsection{Pseudo-ternary phase diagrams}

Pseudo-ternary phase diagrams were used to evaluate the potential of a specific combination of oil/water/emulsifier to form an emulsion and map the region of interest according to composition [52]. Pseudo-ternary phase diagrams were built by covering the entire range of compositions, namely oil (SAO), water and emulsifier (PS or extract (TT, FG, and RA)). Briefly, to prepare the samples (18 different compositions were prepared), the oil and the emulsifier were weighted and mixed for $1 \mathrm{~min}$ using a vortex (V-1 plus Biosan). After, the corresponding weighted water was added and mixed for $5 \mathrm{~min}$. The citric acid $(0.02 \mathrm{wt} \%)$ was added as a preservative, causing a slight decrease on the natural $\mathrm{pH}$ of the emulsions. The assigned type of emulsion for diagrams' construction was based on the visual inspection and macroscopic identification, namely Winsor's Types I, II, III, and IV, and gel-type when the sample was highly viscous. When no emulsion was formed the corresponding points were assigned as "not emulsified". 


\section{Results and discussion}

\subsection{Solubility tests}

The results obtained for solubility in water, ethanol, ethyl acetate, and n-hexane are shown in Table 1 . The methodology presented good reproducibility with a maximum standard deviation of $0.13 \mathrm{~g} / \mathrm{kg}_{\text {solv }}$. For all samples, the solubility (expressed in $\mathrm{g}$ of solute per $\mathrm{kg}$ of solvent) in water, a polar and protic solvent, was high (higher than $85 \%$ ). The solubility in water is in all cases very high, presenting concentration levels much above the CMC, suggesting a mechanism of solubilisation by micellization. [53]. Moreover, according to the Bancroft's rule, this observation corroborates their potential to act as an O/W emulsifier [54, 55]. For solvents of intermediate polarity but holding different nature, ethanol (polar and protic nature) and ethyl acetate (polar and aprotic nature), the samples showed a relatively low solubility, namely within the range from 0.06 to $6.25 \mathrm{~g} / \mathrm{kg}$ solvent. The solubility in ethanol is higher than the one in ethyl acetate, which was associated to its protic nature. The FG extract presented the lowest solubility in organic solvents, namely in ethanol and ethyl acetate, indicating the poorest affinity to hydrophobic solvents. All solutes (PS and extracts) dissolved sparingly in n-hexane. The dominant polar character of saponins, which is derived from their sugar components, hampers the formation of reverse micelles in apolar media, confirming this low solubility.

\subsection{Fourier-transform infrared spectroscopy (FTIR) analysis}

Due to the importance to rationalise the emulsifier potential in terms of structural features, the molecular structure of the four studied samples was analysed by FTIR. Moreover, the soluble fractions recovered during the solubility studies (Section 3.1) were also characterised.

Regarding the chemical composition, saponins are glycosides where the hydrophilic groups comprise sugars such as xylose, galactose, rhamnose, fucose, and derivatives like glucuronic acid, while the characteristic hydrophobic backbone can be either steroidal or triterpene aglycones. Considering the steroidal saponins they usually show furostanol or spirostanol forms. The chemical structure of steroidal and triterpene saponins is shown in Fig. 1. They contain several distinct functional groups (e.g. $-\mathrm{OH},-\mathrm{COOH},-\mathrm{CH}_{3}$ ), which are responsible for the high saponin's diversity. According to reported data, the only ionisable group is the glucuronic acid, since the other acids are attached to the main structure through ester bonds $[17,57]$.

Fig. 2 displays the FTIR spectra of the studied extracts and pure saponin, and Fig. 3 shows the FTIR spectra of each sample in comparison to their respective soluble fractions in water and ethanol (PS (Fig. 3a), TT (Fig. 3b), FG (Fig. 3c), and RA (Fig. 3d)). For PS sample, which was used as reference, a characteristic broad peak centred at $3287 \mathrm{~cm}^{-1}$ due to sugar and acid hydroxyl groups was observed. Other vibrations were assigned at $2930 \mathrm{~cm}^{-1}$ (C-H stretching vibrations), and $1720 \mathrm{~cm}^{-1}$ ( $\mathrm{C}^{=} \mathrm{O}$ stretching attributed to the acid groups of glucuronic, quillaic, and gypsogenic acids). The occurrence of the $\mathrm{C}^{=} \mathrm{C}$ (double-bounds from quillaic acid) was assigned at $1603 \mathrm{~cm}^{-1}$, and the $\mathrm{C}-\mathrm{O}-\mathrm{C}$ stretching vibration derived from the oligosaccharide linkages present in the sugar residues was identified at $1035 \mathrm{~cm}^{-1}$. The observed vibrations were in accordance with the ones previously reported in other works for Quillaja Bark saponin [59].

Table 1

Solubility measurements of pure saponin (PS), and extracts (TT, FG, and RA) in water, ethanol, ethyl acetate, and n-hexane at $30^{\circ} \mathrm{C}$.

\begin{tabular}{lllll}
\hline & \multicolumn{4}{l}{ Solubility $\left(\mathrm{g} / \mathrm{kg}_{\text {solv }}\right) \pm$ standard deviation } \\
\cline { 2 - 5 } & Water & Ethanol & Ethyl acetate & n-Hexane \\
\hline PS & $89.36 \pm 0.05$ & $5.77 \pm 0.11$ & $1.59 \pm 0.07$ & $0.07 \pm 0.00$ \\
TT & $85.40 \pm 0.02$ & $5.93 \pm 0.13$ & $1.75 \pm 0.07$ & $0.53 \pm 0.03$ \\
FG & $87.28 \pm 0.03$ & $0.93 \pm 0.13$ & $0.06 \pm 0.03$ & $0.06 \pm 0.02$ \\
RA & $90.34 \pm 0.10$ & $6.25 \pm 0.01$ & $0.14 \pm 0.06$ & $0.03 \pm 0.00$ \\
\hline
\end{tabular}

The three extracts presented similarities with respect to the pure saponin. They include the peaks assigned to $\mathrm{O}-\mathrm{H}\left(3287 \mathrm{~cm}^{-1}\right), \mathrm{C}-\mathrm{H}$ $\left(2930 \mathrm{~cm}^{-1}\right)$, and C-O-C $\left(1035 \mathrm{~cm}^{-1}\right)$.

Despite the high saponin content ( $~ 93 \%$ ) of TT extract, close to the pure saponin, the spectra showed some differences compared to that of the PS sample. The main variations were reflected in the substantial reduction of the peaks attributed to $\mathrm{C}^{=} \mathrm{O}\left(1720 \mathrm{~cm}^{-1}\right), \mathrm{C}^{=} \mathrm{C}(1603$ and $\left.1513 \mathrm{~cm}^{-1}\right)$ and $\mathrm{C}-\mathrm{O}\left(1235 \mathrm{~cm}^{-1}\right)$ groups. This behaviour could be related to the differences in the two saponins' composition, known to be different according to diverse factors, including their origin or growth conditions. In fact, the saponins can be subdivided into triterpenoid and steroid glycosides, with the latter being formed from spirostan and furostan [8]. PS from Quillaja Bark is characterised for holding triterpene glycosides [13], and TT is generally composed of furostanol glycosides [24], which are characterised by presenting fewer double bonds and oxygens than the triterpenoids. These differences were corroborated by the obtained PS and TT spectra.

Even the lower saponin content in FG $(\sim 50 \%)$ and RA $(\sim 20 \%)$ extracts, a considerable resemblance was found between the FG and TT spectra, which can be attributed to their saponin similar chemical composition, including furostanol glycosides [60]. RA extract showed peaks analogous to the ones of PS spectra (e.g. 1720, 1603, and $1235 \mathrm{~cm}^{-1}$ ), but less intense (supported by the low saponin content) highlighting the presence of triterpenes [43] in its composition.

In general, the spectra of the PS soluble fractions and the extracts in water resulted similar to their original samples, explained by its high solubility in this medium. The increase of the peaks corresponding to the hydroxyl groups in the water-soluble fractions, indicated that the chemical structures holding - $\mathrm{OH}$ groups in the saponins were more prone to be dissolved in water. The ethanol fractions resulted in spectra holding, even slight, some differences. At this point, it is important to highlight the significant differences observed in the dissolution capacity of the samples in water and ethanol (around 85-90, and 5-6\%, respectively). The lower dissolution capacity of the samples in ethanol imply that only some specific molecules were dissolved in ethanol. Thus, in the case of PS, considering their triterpene glycosides rich composition, the disappearance of the peak at $1603 \mathrm{~cm}^{-1}\left(C^{=} C\right)$ suggests the weak capacity of these type of structures to be dissolved in ethanol. In the TT extract, rich in furostanol-based compounds, an increase in the peaks at 2925 and $1382 \mathrm{~cm}^{-1}$, both related to the $\mathrm{C}-\mathrm{H}$ (stretching and bending) modes, was noticed in the recovered ethanolic fractions. In the case of FG and RA, the observed differences in their ethanolic fractions were, in general, in accordance with the behaviour observed for TT and PS fractions, corroborating the similar chemical structure of saponins present in FG and TT (rich in steroid saponins), and present in RA and PS (rich in triterpenoid saponins), as pointed out by the analysis of the spectra of the original compounds.

In the case of FG, the alkaloids are known to have low solubility in water and high in organic solvents, including ethanol, as evidenced by the increase of the peak at $1644 \mathrm{~cm}^{-1}$. Furthermore, it should be worth noting the presence of maltodextrin (MD) in the FG and RA extracts composition. This polymer could also influence the spectra of the samples submitted to different solvents. The MD is highly soluble in water and holds low solubility in ethanol, which could be related with variations in the peak at $1147 \mathrm{~cm}^{-1}$ (FG) and $1143 \mathrm{~cm}^{-1}$ (RA), commonly attributed to the MD [61], whose presence in water fractions disappeared when dissolved in ethanol.

\subsection{Critical micelle concentration assays}

The obtained CMC values were $0.121,0.589,0.904$, and $0.756 \mathrm{~g} \mathrm{~L}^{-1}$ for PS, TT, FG, and RA, respectively, as shown in Fig. 4. These values were determined from the plot of the absorbance against the logarithm of the extract's (or saponin for PS sample) concentration. At the point where the micelles start to form, a sudden rise in absorbance occurs, which is indicated by an arrow in Fig. 4. 


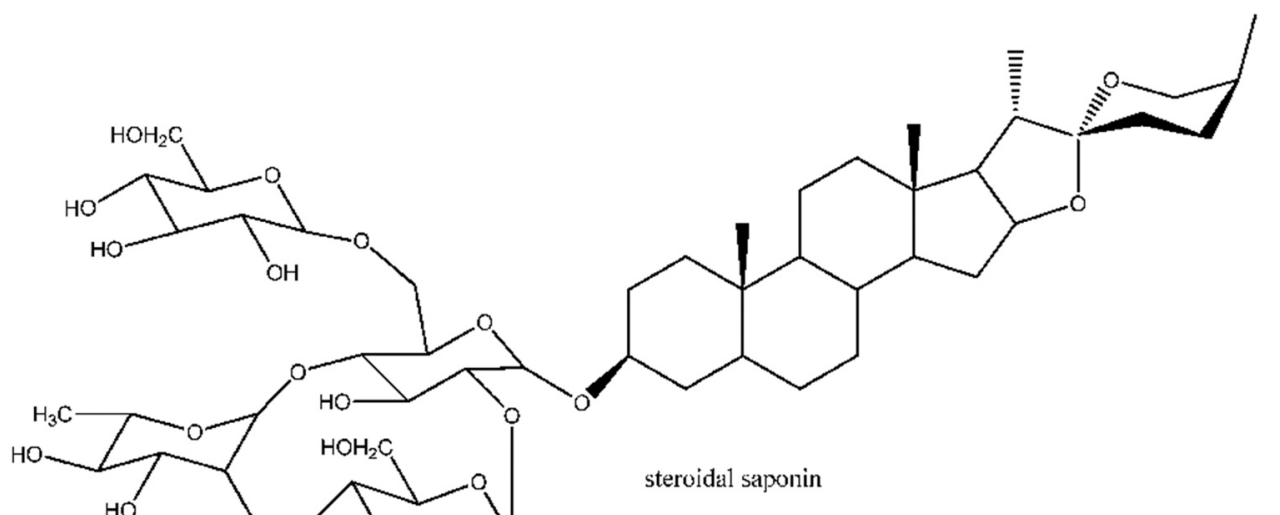

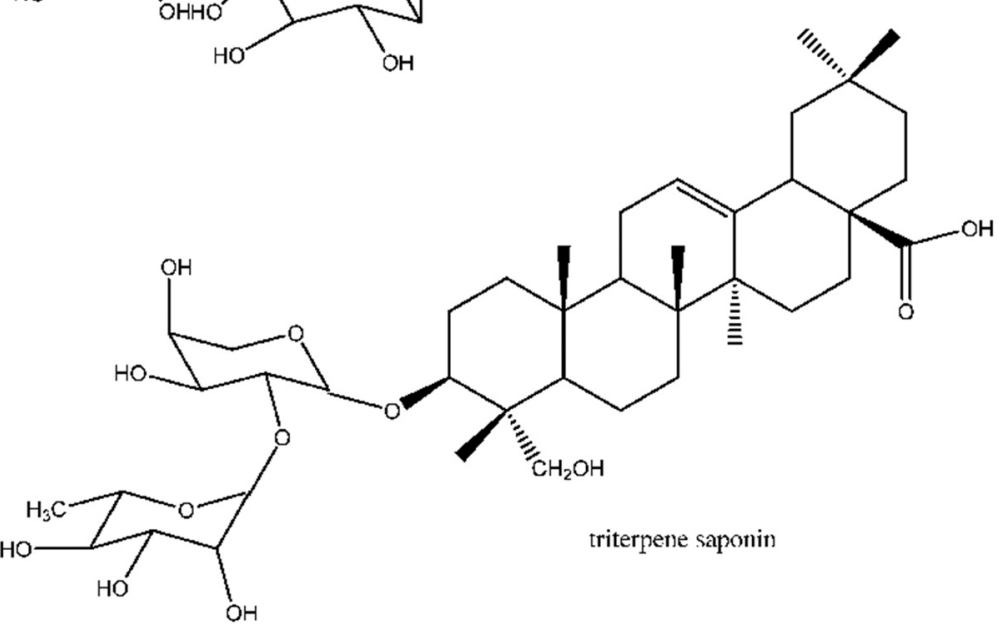

Fig. 1. Steroidal and triterpene saponins examples (monodesmosides with one sugar chain), from Wink [58].

The CMC of quillaja saponin has been reported in the literature by several authors with values ranging between 0.005 and $0.77 \mathrm{~g} \mathrm{~L}^{-1}[56$, 62,63], which includes the value obtained in the present work $\left(0.121 \mathrm{~g} \mathrm{~L}^{-1}\right)$. Regarding the extracts, only a few studies addressing TT extract can be found, being the CMC values in the range comprised between 0.048 and $0.106 \mathrm{wt} \%[16,64]$, which is also in agreement with the value determined in the present work (close to $0.059 \mathrm{wt} \%$ ).

It is well known that as higher as the emulsifier capacity is, the lower its CMC is, demanding less emulsifier to form an emulsion. One result that should be taken into account is that the magnitude of the CMC values did not follow the saponin content, i.e., despite FG extract has a greater saponin content ( $30 \%$ more than RA extract), the respective CMC was higher $\left(0.876\right.$ versus $\left.0.756 \mathrm{~g} \mathrm{~L}^{-1}\right)$, showing to be the extract less efficient in terms of emulsion capacity. This behaviour can be explained by the saponin type present in the extracts, where FG was associated with furostanol glycosides, similarly to TT, and RA to triterpene glycosides like PS, as previously described in Section 3.2.

\subsection{Emulsifying capacity and emulsion stability}

The study of emulsions stability and the ability of saponin-rich extracts to act as emulsifiers was evaluated under different $\mathrm{pH}$ conditions. The mixtures' natural $\mathrm{pH}$ (i.e. without $\mathrm{pH}$ adjustment) was 4.85, 5.00, 5.10, and 4.10 for PS, TT, FG, and RA, respectively. The results of EC and ES for the studied pHs are represented in Fig. 5.

For samples with no $\mathrm{pH}$ adjustment, the results are directly proportional to the saponin content, i.e., the higher the saponin content, the higher the achieved EC values. It is possible to see that although pure saponin presents higher values, reaching $82 \%$, the results for the extracts also showed promising emulsion capacity, agreeing with other studies [65]. Emulsions' stability also presented satisfactory outcomes.
Regarding $\mathrm{pH}$, it has a strong impact on emulsion formation and stability since it influences the electrostatic repulsion between droplets. According to the DLVO theory, the energy barrier is responsible for inhibiting droplets from getting close to each other to aggregate. If the electrostatic repulsion decreases, it is more difficult to avoid droplet's aggregating [66]. The acid medium ( $\mathrm{pH} 3$ ) benefited the extracts' capacity to form emulsions, leading to the best EC results if compared with their natural pH. For RA, for example, the value of EC went from $36 \%$ $(\mathrm{pH}=4.1)$ to $49 \%(\mathrm{pH}=3)$. This behaviour can be an advantage for specific applications in this range of $\mathrm{pH}$. Pure saponin showed a pronounced decrease, reaching approximately $66 \%$. In the same $\mathrm{pH}$, the stability (ES) showed two different responses, while for PS and TT, there is an increase in its value; for FG and RA, the stability decreased, which can be explained by the saponin content. The presence of other functional groups (flavonoids and phenolic compounds) might have interfered with the emulsion stability [22]. This behaviour has been hypothesised in other published works where some mechanisms have been proposed to describe the interference of polyphenols in lipid's emulsification. These effects include interactions between the hydroxyls of polyphenols (or flavonoids) with other hydrophilic/polar groups present in the media. Their possible incorporation in the emulsified layer, leading to the emulsion physicochemical properties change, was also described [67].

At neutral $\mathrm{pH}$, PS presented the best results both for the capacity and stability, being this last one even higher than at natural $\mathrm{pH}$. This behaviour was shown before for $\mathrm{pH} 7$ [68], and can be explained by the deprotonation of the carboxylic acid groups, increasing the negative charges in the saponin molecules and their stability [69]. However, the extracts exhibit different behaviours, with RA, the extract with the lower saponin content, presenting higher values than FG, both in emulsion capacity and stability. The total amount of saponin on its own is not 


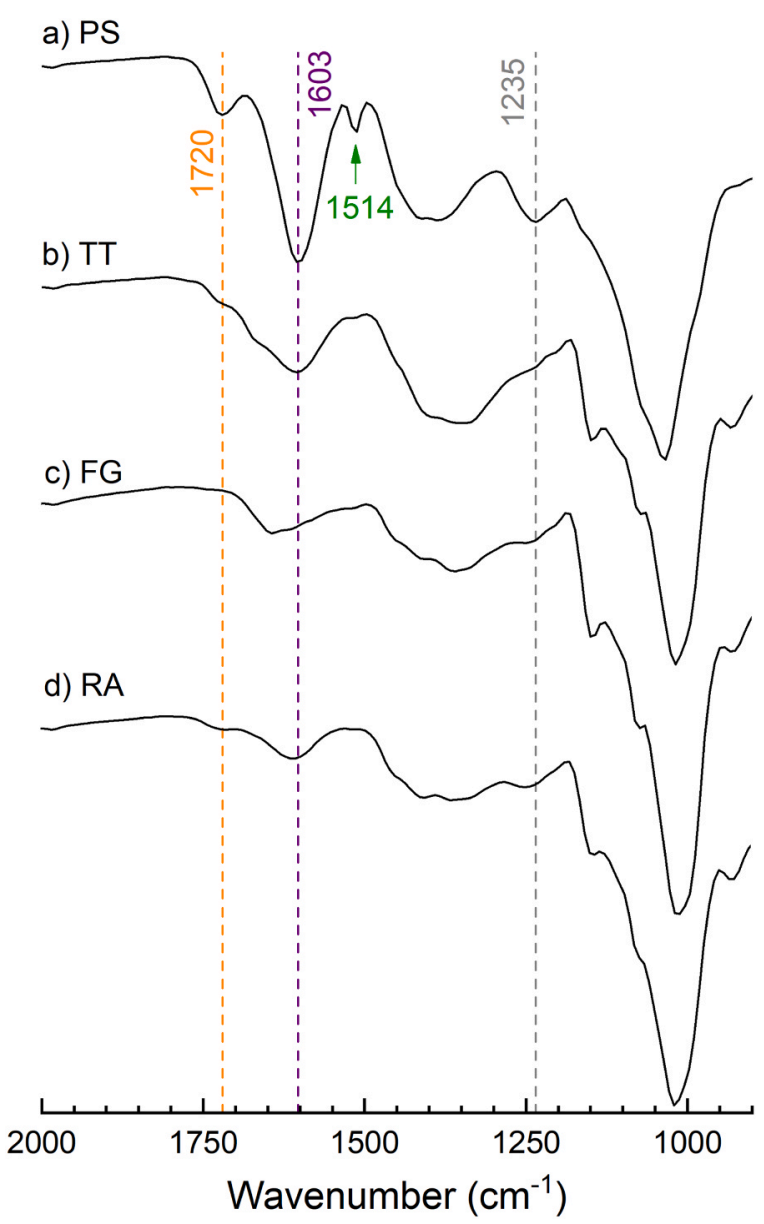

Fig. 2. FTIR spectra of PS (a), TT (b), FG (c), and RA (d) samples.

enough to explain the emulsifying power; for example, TT extract presents a high amount of saponins but presents much less emulsifying power than pure saponin. Possibly this is related to have steroidal type saponins in TT instead of triterpenic as it happens in pure saponin [64]. At basic $\mathrm{pH}(\mathrm{pH}$ 9), the values of EC for the four samples (pure saponin and extracts) was similar, with RA presenting the highest EC ( 54\%). The basic media may promote the hydrolysis of the glycosidic and ester bonds, leading to a decrease in its performance as emulsifier [70,71]. Some possible scenarios include the emulsion tendency to flocculation, the attraction of cationic multivalent ions and/or pro-oxidants, and the adhesion of the droplets to cationic surfaces [5]. This effect was observed for TT, but particularly for PS, whose relative higher amount of $\mathrm{COOH}$ groups in comparison to the studied extracts (as evidenced by FTIR results), strengthened the phenomena. For the ES results, the sequence based on the saponin content was verified.

Based on the previous results, it was possible to observe that, in general, the thumb rule is, the higher is the amount of saponin, the more efficient the extract is as an emulsifier. However, in some $\mathrm{pH}$ media, this behaviour was not observed. At $\mathrm{pH}$, the EC values of the four samples become close, meaning that they have similar emulsifying capacity. The performance of RA was slightly superior than FG for $\mathrm{pH} 7$ and 9. This behaviour was also predicted by the conductivity measurements. In fact, the presence of other compounds can help or hinder the activity of extracts in terms of emulsifying capacity and emulsion stability. Besides, comparing the PS and TT (extract with better EC and ES performance), the $\mathrm{pH}$ media strongly affected the PS, with an EC variation of approximately $31 \%$, whereas TT showed more resistance, with a variation of only $7.5 \%$.

The optical microscopy analysis allows a better visualisation of the emulsions' morphology. Fig. 6 shows the images acquired at the tested pHs. The number of the observed droplets per volume unit is directly related to the ability to form an emulsion and its stability, i.e., some samples present fewer droplets because part of the oil phase was destabilised, forming an oil layer at the top of the system, implying that the analysed emulsion had a lower oil content. This fact means that more droplets are visible in the emulsion as the EC and ES values increase. Comparing the four emulsifiers, this behaviour can be easily perceptible in the first column of Fig. 6 (no pH adjustment), presenting the sample prepared with pure saponin more droplets (also the highest EC), followed by TT, FG, and then RA. An intriguing feature from Fig. 7 is that RA, even with lower EC and ES, shows the smallest droplets, which seems contradictory. This system showed some phase segregation since larger and less stable droplets induced creaming, while some of the smallest droplets remained in the bottom phase (emulsion).

For the samples prepared at $\mathrm{pH} 3$, and the extracts FG and RA, an increase in the droplet size was observed, comparatively with the samples prepared with no $\mathrm{pH}$ adjustment. This observation is consistent with the decrease in the emulsion stability (Fig. 5). For PS and TT, a slight decrease in the droplet size, also in accordance with results of emulsion stability, was verified. As evidenced by the EC results, $\mathrm{pH} 9$ is the best condition for RA emulsions' formation and stability, even better than its natural $\mathrm{pH}$. The same conclusion is evident from the microscopy analysis, where the droplets presented round shape and higher homogeneity. Images of the emulsions prepared at $\mathrm{pH} 9$ showed the most significant change from the ones prepared with no $\mathrm{pH}$ adjustment. Moreover, the round shape indicates better stability. A comparison of the tested extracts shows the highest similarity among samples, following the previous results found for EC and ES.

Another important topic is the size heterogeneity of droplets that can be explained by the used method (mechanical stirring), which can be solved with further processing.

\subsection{Foaming properties}

Surface-active compounds are responsible for foam formation and stability. These systems are generated, typically, by mechanical stirring and are thermodynamically unstable [72]. In this work, foaming properties were identified for the four tested samples to find the ones more suitable for applications requiring high or low foaming characteristics. The values of foaming capacity (FC) and foaming stability (FS) are found in Table 2.

It can be seen that the FC and FS values of PS are considerably higher (up to $390 \%$ ) than the ones of the extracts (TT, FG, and RA), which are similar among them, ranging from $112 \%$ to $130 \%$. Two-way ANOVA was applied at a 5\% significance level to evaluate statistically significant effects; $\mathrm{pH}$ and saponin source on the foam formation and stability. In both cases, the interaction between the two effects are very significant (p-value lower than 1E-5), not recommending the analysis of the individual effects separately. This fact can be considered an advantage of saponin-rich extracts over the pure saponin for cases where it is not interesting for the final product to present a high foam amount. This is the case of cosmetics (e.g. creams, face masks, and make-up) [73]. Moreover, the excess of foam can be harmful to processes like high-pressure homogenisation [74], and the equipment can be damaged [75]. Despite the results of extracts being very close, RA shows higher foam formation and stability at higher $\mathrm{pH}$. The saponin type can explain this outcome, where RA contains in its structure the triterpene glycosides, known as foaming agents [70].

In a general way, it was noticed a good foam stability for all the tested foams, in particular for PS. Again, the samples subjected to $\mathrm{pH} 3$ were the ones with the lowest foam stability, except for TT that only showed a variation of $4.2 \%$ in the foam volume. Under the tested $\mathrm{pHs}$, FG extract was the one with the lowest stability among the tested samples, losing on average $7.7 \%$ of its initial foam, which can be assigned to its saponin type (steroidal) and composition [24]. 
a) PS

Reference
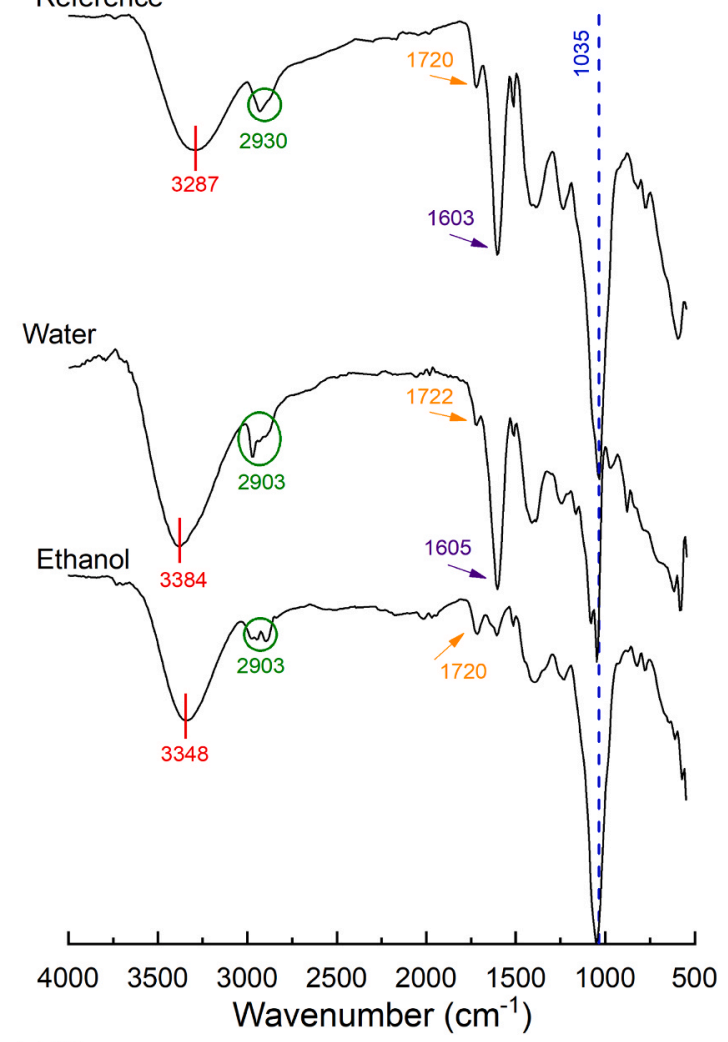

c) FG

Reference<smiles>CCCC</smiles>

3287

Water

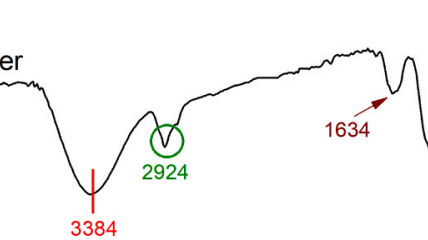

3384

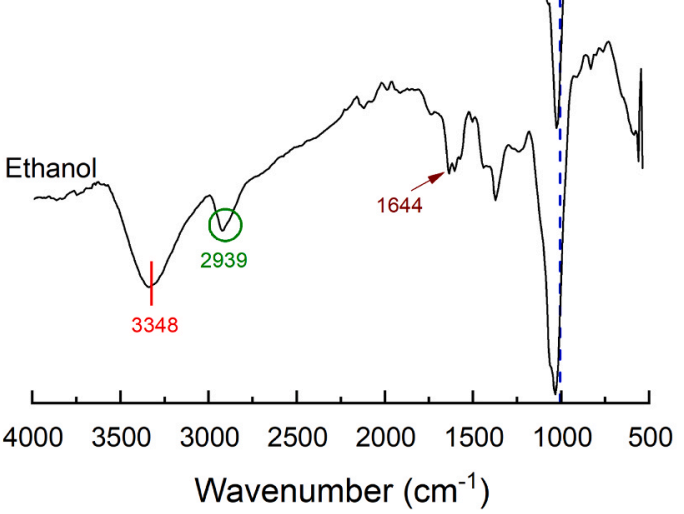

b) $\mathrm{TT}$

\section{Reference}

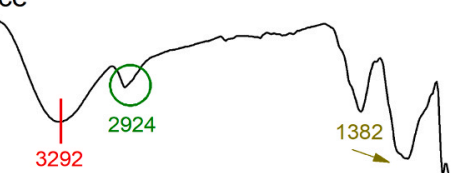

$\stackrel{\infty}{\stackrel{\circ}{\circ}}$

$1 \div$

is $M$
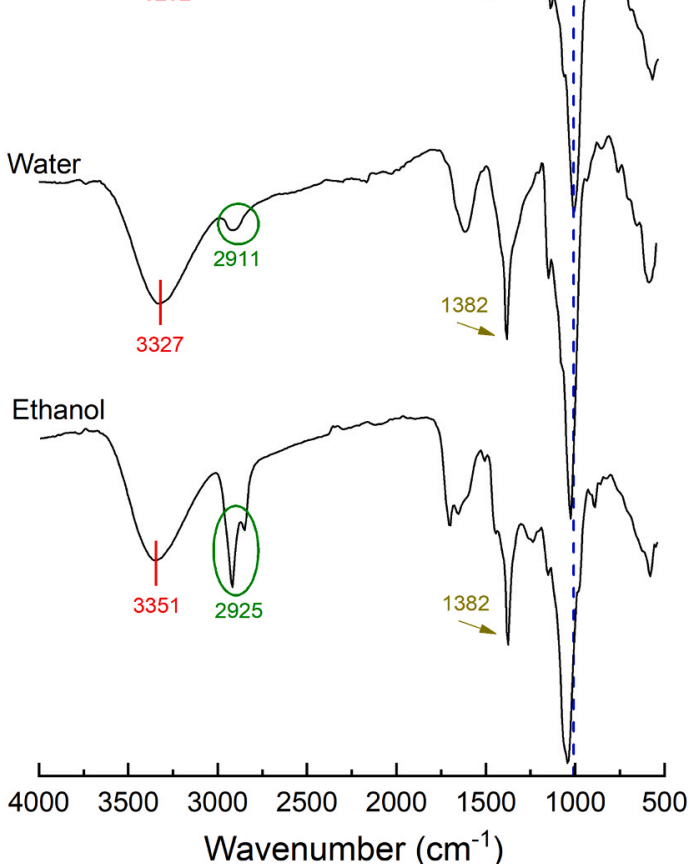

d) $R A$

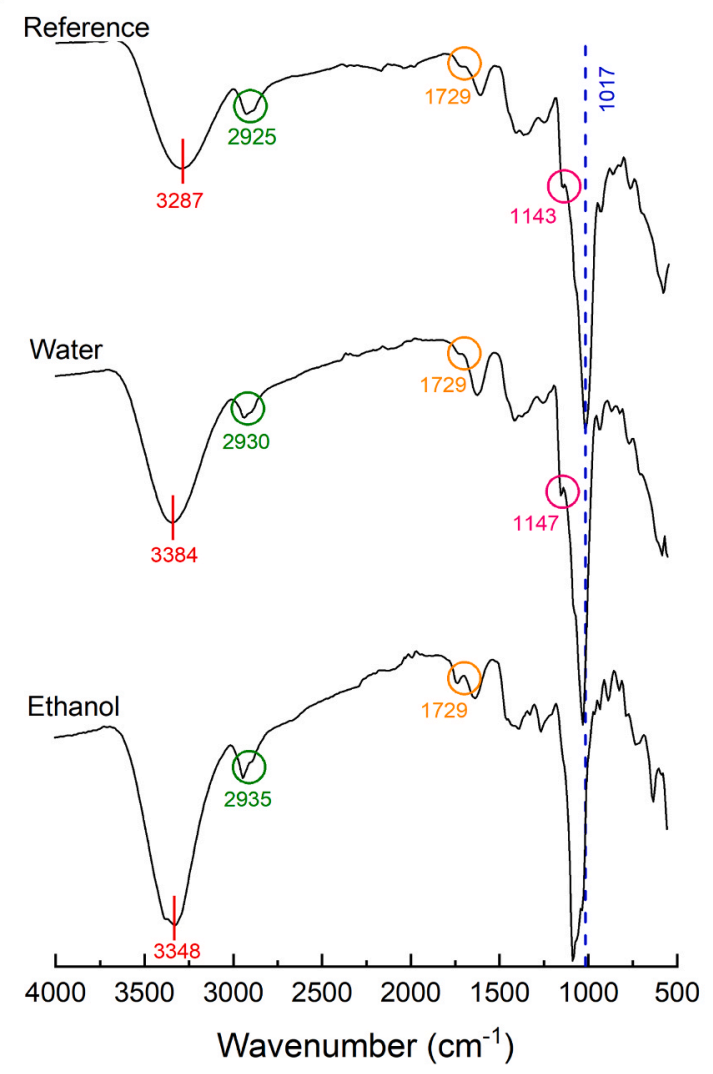

Fig. 3. FTIR spectra of PS (a), TT (b), FG (c), and RA (d) samples, and their respective soluble fractions in water and ethanol. 


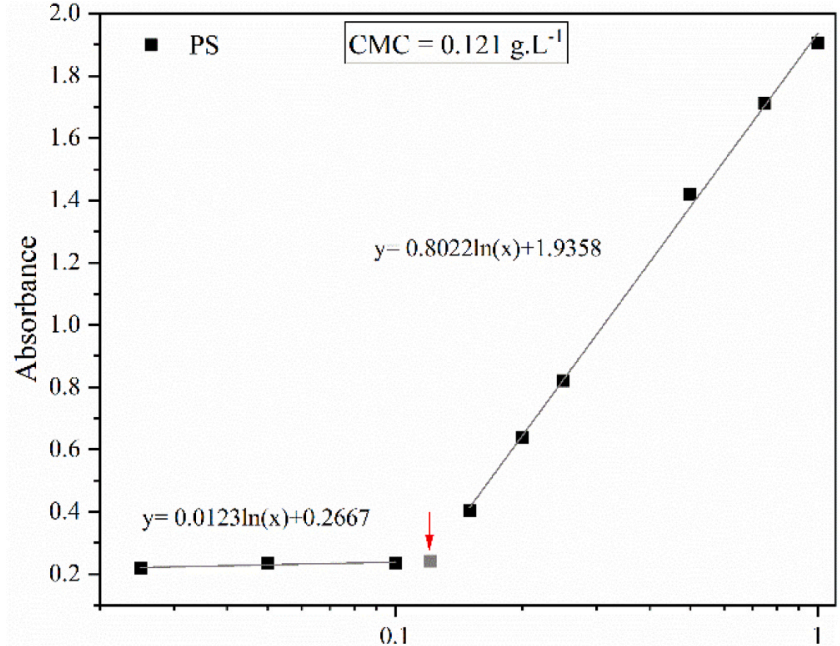

Concentration $\left(\mathrm{g} \cdot \mathrm{L}^{-1}\right)$

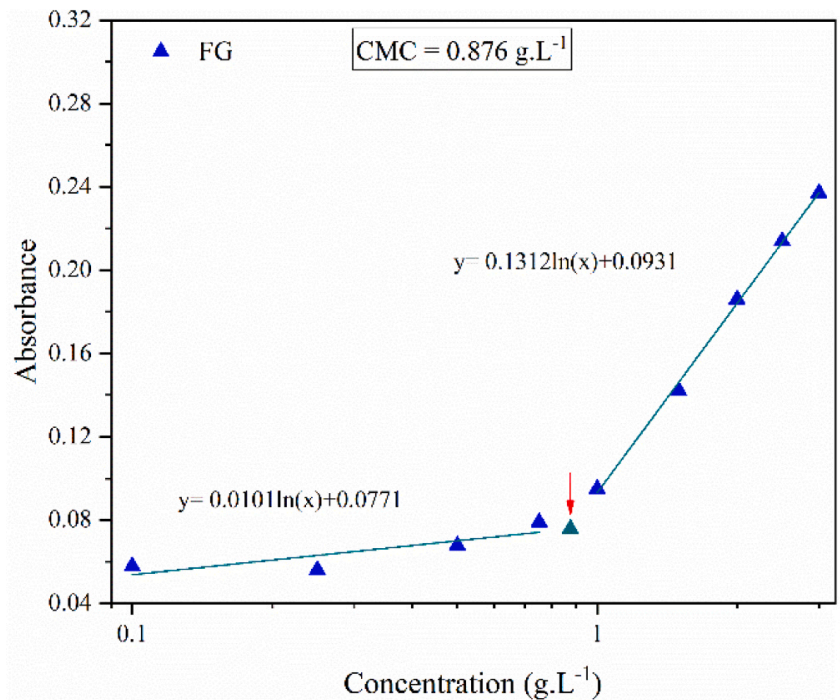

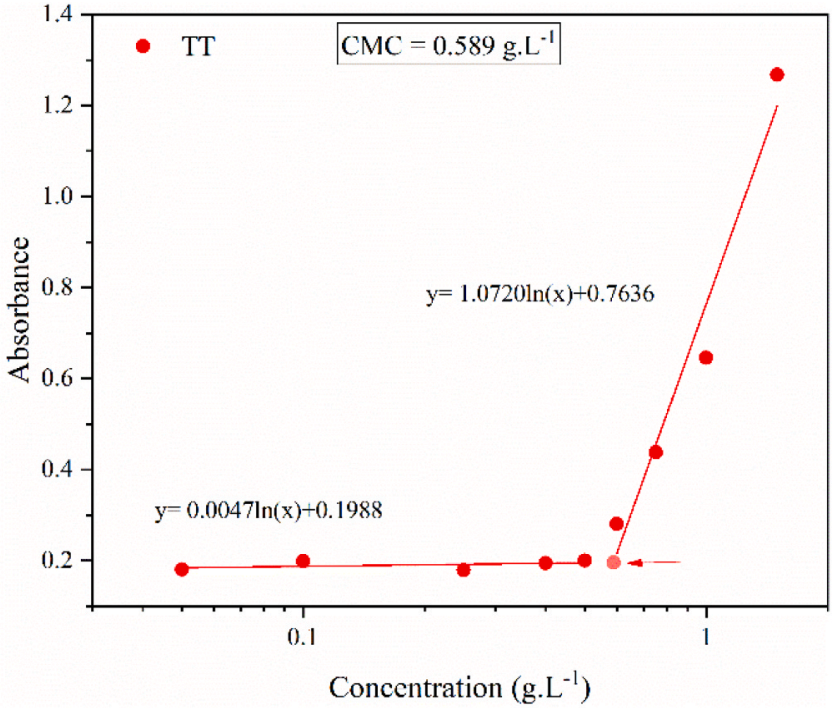

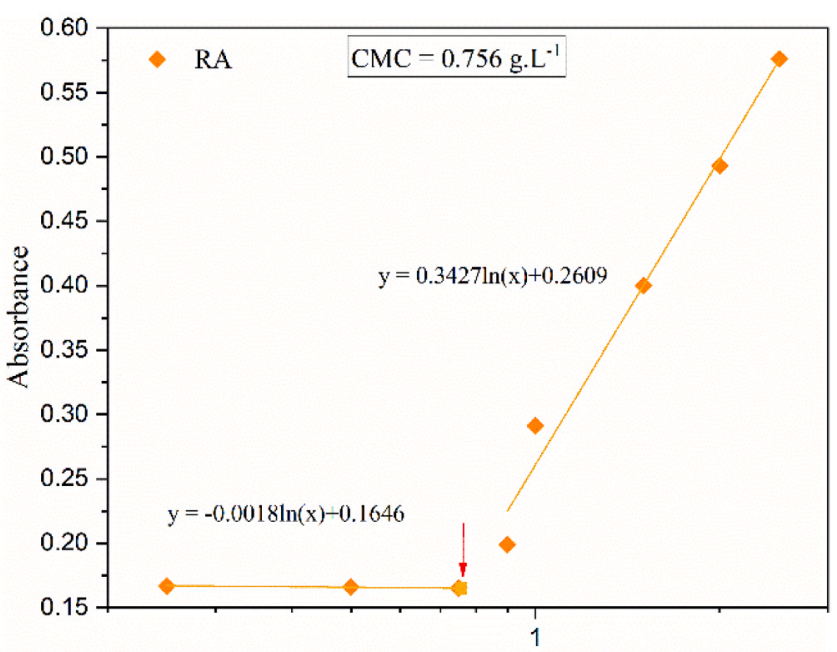

Concentration $\left(\mathrm{g} \cdot \mathrm{L}^{-1}\right)$

Fig. 4. Graphical representation of the absorbance as a function of PS, TT, FG, and RA concentration (logarithmic scale). CMC, which corresponds to the interception of the two zones described by the included linear relationships is assigned by an arrow.
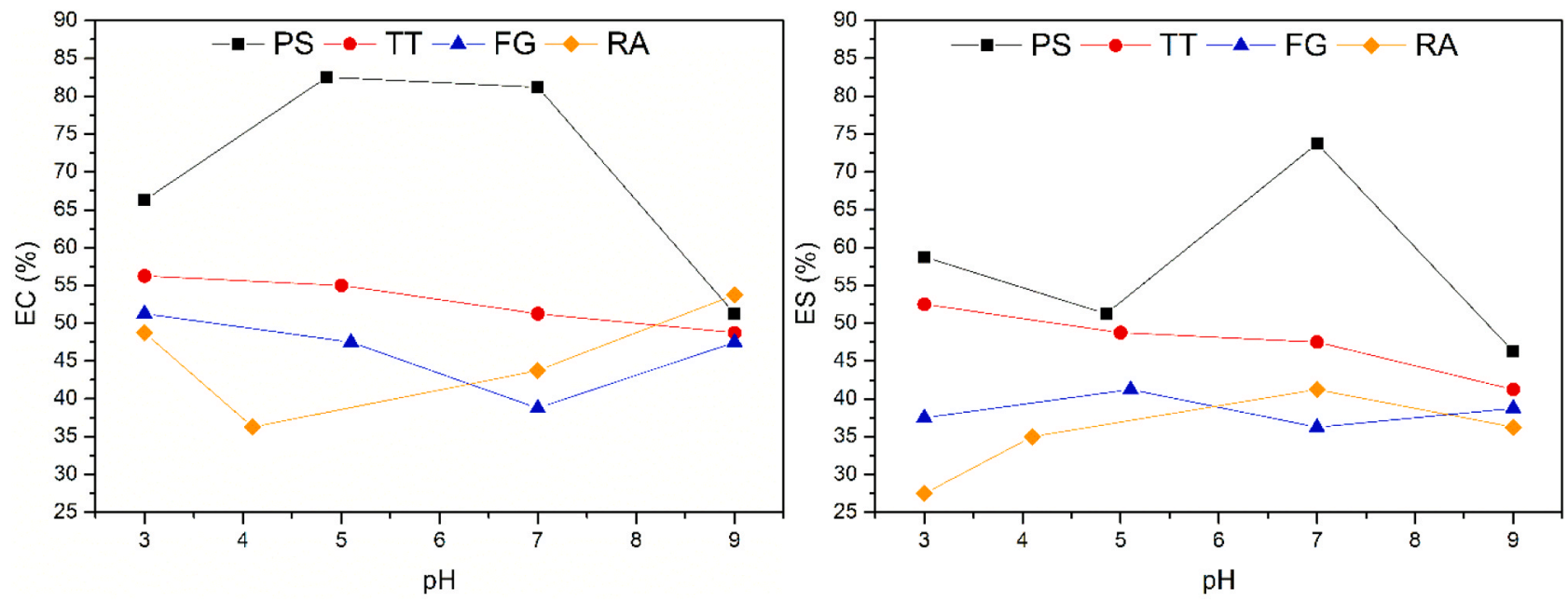

Fig. 5. Comparative emulsifying capacity (EC) and emulsion stability (ES) values at different pHs for the studied samples (PS, TT, FG, and RA). 


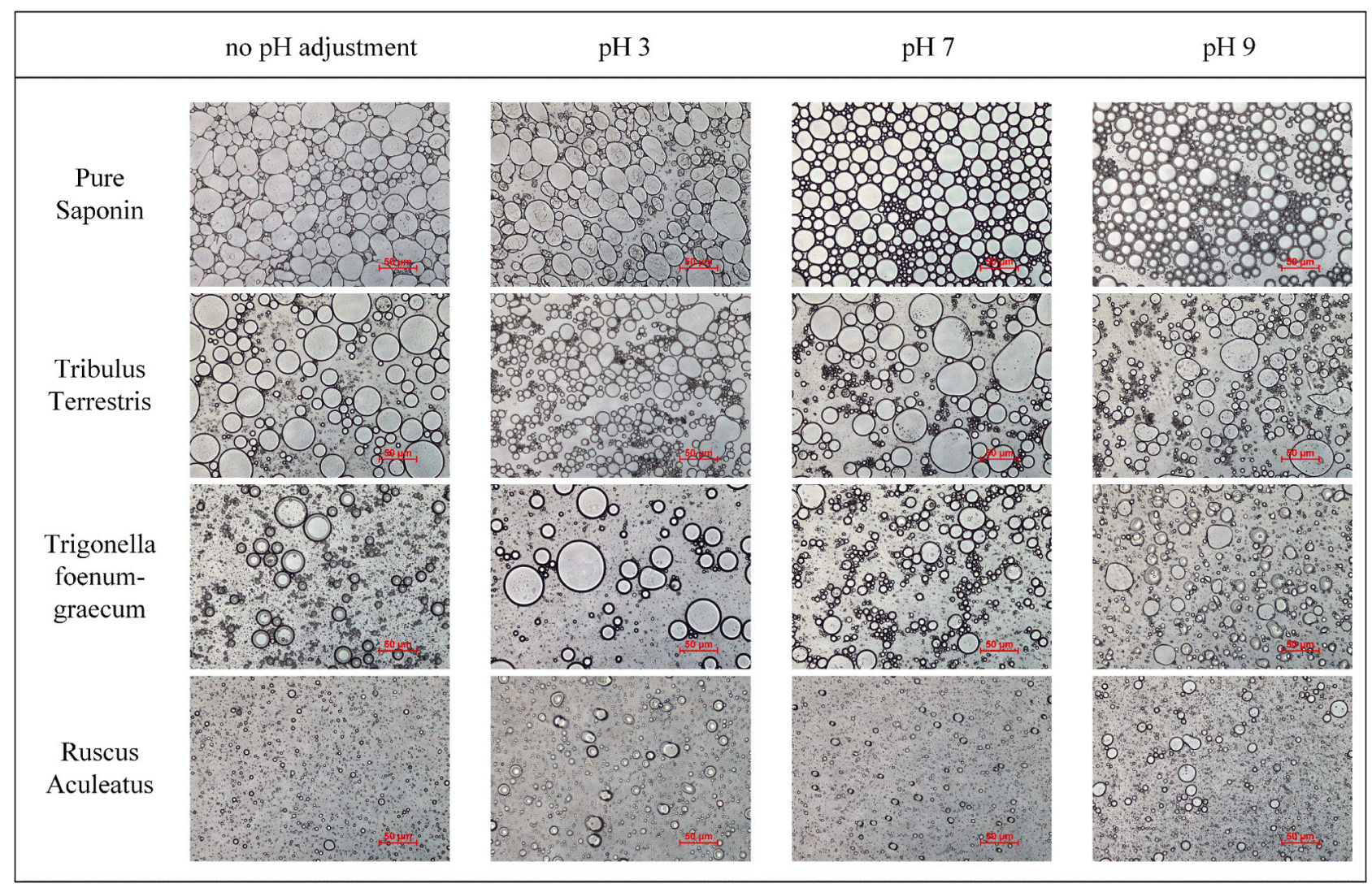

Fig. 6. Optical microscopy of the prepared emulsions at different pHs. Scale bar of $50 \mu \mathrm{m}$.

\subsection{Pseudo-ternary phase diagrams}

Pseudo-ternary phase diagrams were constructed for the four studied natural emulsifiers (PS, TT, FG, and RA). The $\mathrm{pH}$ of the samples was close to the natural emulsions $\mathrm{pH}$ as defined in the emulsifying capacity and emulsion stability studies (4.85, 5.00, 5.10, and 4.10 for PS, TT, FG, and RA, respectively). The categorisation of the produced samples followed Winsor's classification for emulsions [76]. Type I corresponds to a biphasic system, where one of the phases is the oil and the other one an emulsion of oil in water (o/w) type. For this type of emulsion to occur, the emulsifier is usually water-soluble. Winsor's Type II also corresponds to a biphasic system, with the emulsion phase being water in oil (w/o) type. In this case, the second phase (aqueous part) is positioned at the bottom. Type III is a three-phase system, where the emulsified phase is in equilibrium with the excess of free oil and water. The top contains the oil phase, the emulsion is the intermediary phase, and the aqueous phase is at the bottom. The last one, Type IV, corresponds to the most interesting system since it corresponds to a homogeneous and monophasic system, i.e. an emulsion formed between the oil, water, and stabilised by the emulsifier. Fig. 7 shows the resultant pseudo-ternary diagrams for PS, and extracts (TT, FG, and RA) (Fig. 7a-d, respectively).

The formation of single-phase systems (Winsor's Type IV) is observed in the region of low oil and high emulsifier content. This behaviour indicates the tendency of this system to form oil in water $(\mathrm{o} / \mathrm{w})$ emulsions, which has been demonstrated before for PS [13]. Depending on the emulsifiers, the outcomes were slightly different. The PS, TT, and FG showed better performance (that is, broader Winsor's type IV area) than the RA, which can be related to the saponin content. Moreover, in opposition to the previous analysis concerning EC, ES, FC, and FS properties, when compared to the reference PS, in this case, results are much more similar, evidencing thee extracts potential to be used as emulsifiers. For example, TT showed a wider single-phase area (red points) compared to the PS.

Biphasic systems were also observed for all emulsifiers, being formed, in all cases, an o/w emulsion with an excess of oil on the top. This behaviour can be assigned to the small amount of emulsifier used in these formulations, not enough to disperse and stabilise the oil content. The extension of the area presenting this behaviour in the diagrams of the extracts decreases according to the sequence RA $>$ FG $>$ TT, evidencing an inverse correlation to their saponin content. Also, the low solubility of the extracts in less polar solvents indicates their less affinity to the oil phase [77] implying that greater quantities would be required to effectively reduce the interfacial tension between both phases, leading in this case, to the formation of the free oil phase.

The diagram also showed a region where high viscous emulsions, named gel samples, were formed. Indeed, Reichert et al. [78] reported that saponins, under specific conditions, can form gel systems. These systems might be of high interest in terms of physicochemical properties, but with particular attention in their mechanical behaviour due to their ability to reach the gel state without adding thickeners, of great potential in topical applications [79]. In this work, this region was found with a water mass content comprised between $15 \%$ and $45 \%$ and an emulsifier content above $40 \%$. The number of gel-type samples (blue points) in the diagram follows the saponin content sequence, being PS > TT $>$ FG $>$ RA, where for RA, no samples of this type were detected, probably related to the very low saponin content.

Although all the studied extracts present emulsion capability, some samples were not able to emulsify (purple points). This is located at the low water content region; on the left because of unbalanced amounts of oil and emulsifier, and on the right due to the too high extract quantity and low liquid content, hindering the total dissolution to produce the emulsion. 


\section{$\square$ Winsor's Type I $\square$ Winsor's Type IV $\square$ Gel $\square$ Not emulsified}

a)

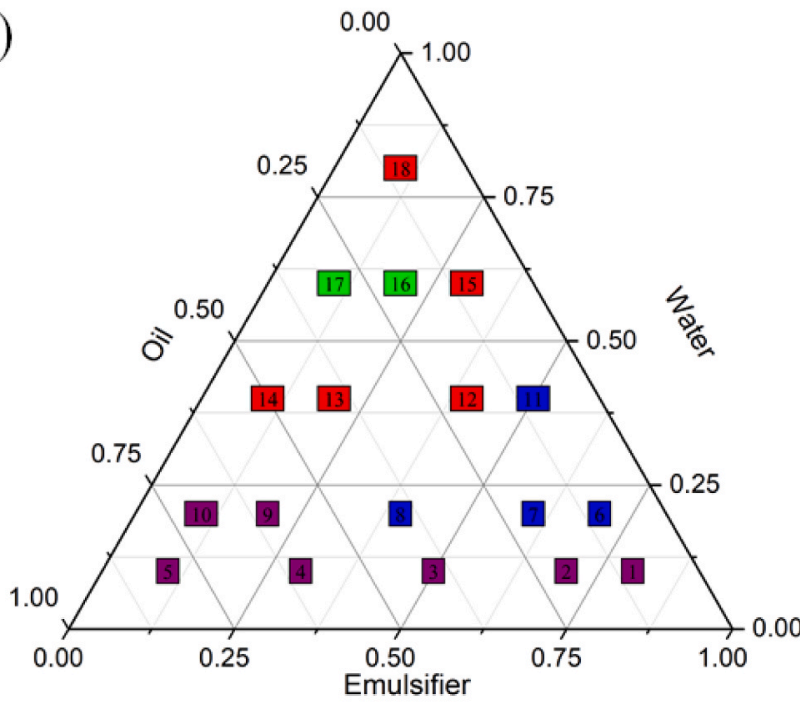

c)

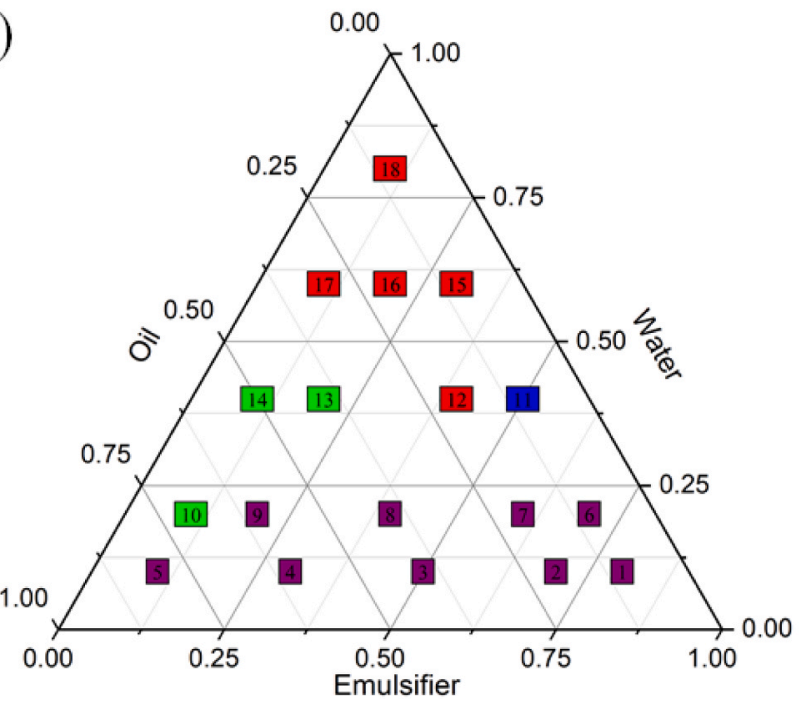

b)

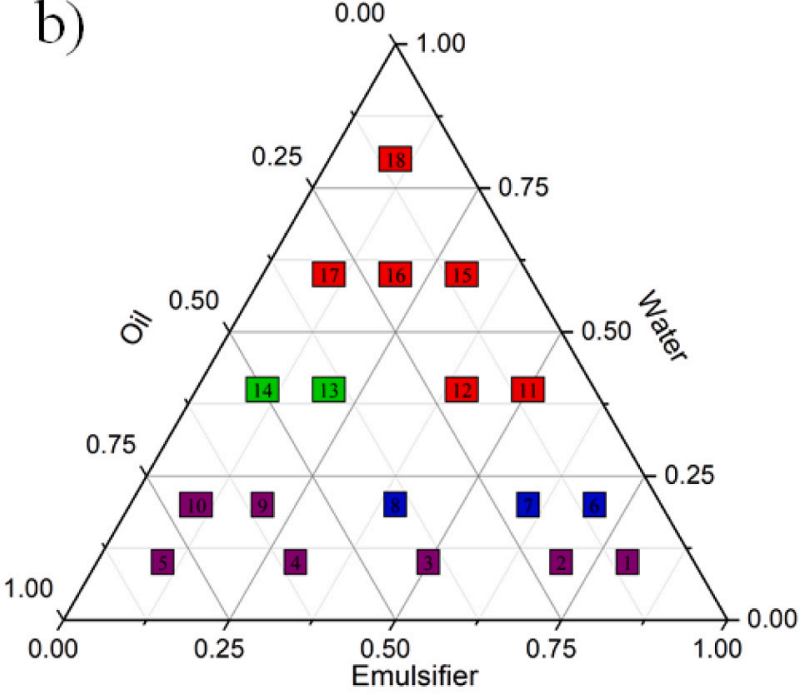

d)

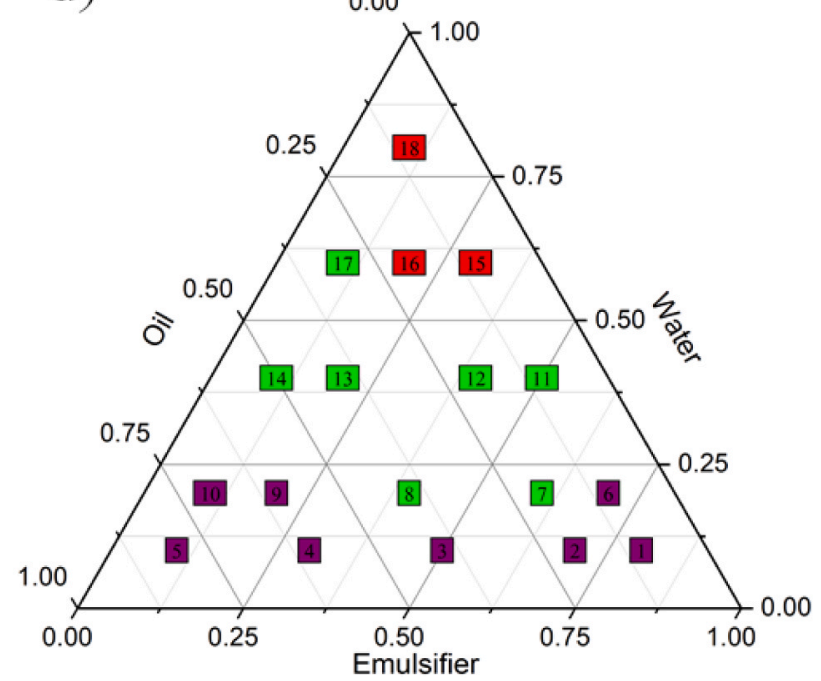

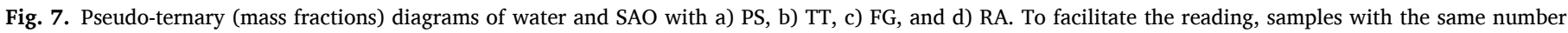
indicate the same composition.

Table 2

Foaming capacity (FC), and foaming stability (FS) in percentage for different pHs.

\begin{tabular}{|c|c|c|c|c|c|c|c|c|}
\hline & \multicolumn{2}{|c|}{ No $\mathrm{pH}$ adjustment } & \multicolumn{2}{|l|}{ pH 3} & \multicolumn{2}{|l|}{ pH 7} & \multicolumn{2}{|l|}{ pH 9} \\
\hline & FC (\%) & FS (\%) & FC (\%) & FS (\%) & FC (\%) & FS (\%) & FC (\%) & FS (\%) \\
\hline PS & $376.9 \pm 2.7$ & $364.3 \pm 6.1$ & $375.5 \pm 3.5$ & $342.5 \pm 3.5$ & $390.0 \pm 7.1$ & $377.5 \pm 3.5$ & $372.5 \pm 3.5$ & $358.0 \pm 2.8$ \\
\hline TT & $129.7 \pm 2.3$ & $122.1 \pm 1.3$ & $120.0 \pm 0.7$ & $115.0 \pm 0.0$ & $120.5 \pm 0.7$ & $116.5 \pm 2.1$ & $126.0 \pm 1.4$ & $121.0 \pm 1.4$ \\
\hline FG & $122.1 \pm 1.3$ & $113.1 \pm 2.7$ & $124.8 \pm 0.4$ & $114.0 \pm 1.4$ & $121.5 \pm 2.1$ & $112.5 \pm 3.5$ & $122.5 \pm 3.5$ & $113.5 \pm 2.1$ \\
\hline RA & $126.8 \pm 0.7$ & $120.2 \pm 1.4$ & $126.0 \pm 1.4$ & $115.5 \pm 1.7$ & $130.0 \pm 0.0$ & $127.0 \pm 2.8$ & $130.0 \pm 0.0$ & $125.5 \pm 0.7$ \\
\hline
\end{tabular}

\section{Conclusions}

In this work, the performance of three saponin-rich extracts (Tribulus terrestris, Trigonella foenum-graecum, and Ruscus Aculeatus) as potential natural emulsifiers was carried out. Their emulsifying capacity, emulsion stability and CMC were compared with the one of pure Quillaja bark saponin, the most commonly used commercial form. This study was complemented with the evaluation of extract's solubility in a range of solvents (water, ethanol, ethyl acetate, and n-hexane), selected to cover different polarities, and the soluble factions examined by FTIR and compared with original sample. To complete the study, pseudo-ternary diagrams were constructed to have a first insight into the composition 
regions of interest for emulsions' preparation and identify other interest systems (e.g. gel-like samples).

The solubility studies revealed that extract's solubility followed the order: water $>$ ethanol $>$ ethyl acetate $>$ n-hexane, in accordance with pure saponin sample. The solubility in water lead to concentration levels much above the CMC, suggesting a mechanism of solubilisation by micellization. Moreover, the high compatibility with water, which can derived also from the presence of compounds other than saponins, corroborate, especially in the cases of high-content saponin samples, the potential $\mathrm{O} / \mathrm{W}$ stabilisation character of these amphiphilic molecules, systems whose composition was validated by the constructed pseudoternary diagram studies. In general, the FTIR analysis pointed out the similarity of FG and TT (rich in steroid saponins), and RA and PS (rich in triterpenoid saponins, even RA can also have steroid-type saponins).

The obtained CMC values were $0.121,0.589,0.904$, and $0.756 \mathrm{~g} \mathrm{~L}^{-1}$ for PS, TT, FG, in accordance with available data published in literature. Moreover, CMC values did not follow the saponin content, which can be related with the presence of other compounds in the studied extracts. EC and ES pointed out the viability of the extracts to act as surfactants, with TT ( 90\%) being highlighted as the most promising extract. Extract's behaviour showed to be dependent on the $\mathrm{pH}$ media, and when compared to PS, they showed lower foam capacity, which might be advantageous for several processes and products, e.g. processing by high pressure homogenisation.

Overall, the obtained results positioned saponin-rich extracts as potential emulsifiers, offering advantages over saponin pure forms, holding similar or even additional functional properties, while avoiding complex extraction and purification treatments. The obtained results pointed out the interest to proceed with these studies, with relevance for a deeper understanding of the interference of extract compounds in the emulsion's technological and function properties, which demand the full chemical and biological characterisation of the samples and the study of their individual and combined/synergistic behaviour.

\section{CRediT authorship contribution statement}

Tatiana B. Schreiner: Conceptualization, Methodology, Investigation, Formal analysis, Writing - original draft, Writing - review \& editing. Giovana Colucci: Methodology, Investigation. Arantzazu Santamaria-Echart: Methodology, Validation, Writing - original draft, Writing - review \& editing. Isabel P. Fernandes: Methodology, Investigation, Writing - original draft. Madalena M. Dias: Conceptualization, Supervision, Writing - review \& editing. Simão P. Pinho: Conceptualization, Supervision, Writing - review \& editing, Resources. Maria Filomena Barreiro: Conceptualization, Supervision, Writing - review \& editing, Resources.

\section{Declaration of Competing Interest}

The authors declare that they have no known competing financial interests or personal relationships that could have appeared to influence the work reported in this paper.

\section{Acknowledgements}

This work was financially supported by: Base Funding - UIDB/ 00690/2020 of CIMO - Centro de Investigação de Montanha - funded by national funds through FCT/MCTES (PIDDAC), Base Funding - UIDB/ $50020 / 2020$ of the Associate Laboratory LSRE-LCM - funded by national funds through FCT/MCTES (PIDDAC), and project AIProcMat@N2020 - NORTE-01-0145-FEDER 000006 supported by NORTE 2020 under the Portugal 2020 Partnership Agreement, through ERDF. National funding by FCT, Foundation for Science and Technology, through the individual research Grant 2020.05564. BD of Tatiana La Banca Schreiner. FCT, PI, through the institutional scientific employment program-contract for I.P. Fernandes contract through the celebration of program-contract foreseen in No. 4, 5 and 6 of article $23^{\circ}$ of Decree-Law No. 57/2016, of 29th August, amended by Law No. 57/ 2017, of 19th July.

\section{References}

[1] C. Qian, D.J. Mcclements, Formation of nanoemulsions stabilized by model foodgrade emulsifiers using high-pressure homogenization: factors affecting particle size, Food Hydrocoll. 25 (2011) 1000-1008, https://doi.org/10.1016/j. foodhyd.2010.09.017.

[2] S. Rebolleda, M.T. Sanz, J.M. Benito, S. Beltrán, I. Escudero, M.L.G. San-josé, Formulation and characterisation of wheat bran oil-in-water nanoemulsions, Food Chem. 167 (2015) 16-23, https://doi.org/10.1016/j.foodchem.2014.06.097.

[3] D.J. McClements, L. Bai, C. Chung, Recent advances in the utilization of natural emulsifiers to form and stabilize emulsions, Annu. Rev. Food Sci. Technol. 8 (2017) 205-236, https://doi.org/10.1146/annurev-food-030216-030154.

[4] D.J. Mcclements, C.E. Gumus, Natural emulsifiers - biosurfactants, phospholipids, biopolymers, and colloidal particles: molecular and physicochemical basis of functional performance, Adv. Colloid Interface Sci. 234 (2016) 3-26. 〈https:// www.sciencedirect.com/science/article/abs/pii/S0001868616300380 .

[5] Y. Yang, M.E. Leser, A.A. Sher, D.J. McClements, Formation and stability of emulsions using a natural small molecule surfactant: Quillaja saponin (QNaturale $($ )), Food Hydrocoll. 30 (2013) 589-596, https://doi.org/10.1016/j. foodhyd.2012.08.008.

[6] S. Jangra, B. Sharma, R. Jangra, V. Chhokar, S. Duhan, Saponin-loaded SBA-15: release properties and cytotoxicity to Panc-I cancer cells, J. Porous Mater. 25 (2018) 945-953, https://doi.org/10.1007/s10934-017-0506-y.

[7] C. Chung, A. Sher, P. Rousset, E. Andrew, D. Julian, Formulation of food emulsions using natural emulsifiers: utilization of quillaja saponin and soy lecithin to fabricate liquid coffee whiteners, J. Food Eng. 209 (2017) 1-11. 〈https://www.sc iencedirect.com/science/article/abs/pii/S0260877417301516).

[8] D. Kregiel, J. Berlowska, I. Witonska, H. Antolak, C. Proestos, M. Babic, L. Babic, B. Zhang, Saponin-based, biological-active surfactants from plants, in: Application and Characterization of Surfactants, InTechOpen, 2017, pp. 183-205, https://doi. org/DOI: 10.5772/68062.

[9] Ö. Güçlü-Üstündag, G. Mazza, Saponins: properties, applications and processing, Crit. Rev. Food Sci. Nutr. 47 (2007) 231-258, https://doi.org/10.1080/ 10408390600698197.

[10] T. Pekdemir, M. Çopur, K. Urum, Emulsification of crude oil-water systems using biosurfactants, Process Saf. Environ. Prot. 83 (2005) 38-46, https://doi.org/ 10.1205/psep.03176.

[11] S. Uluata, D.J. McClements, E.A. Decker, Physical stability, autoxidation, and photosensitized oxidation of $\omega-3$ oils in nanoemulsions prepared with natural and synthetic surfactants, J. Agric. Food Chem. 63 (2015) 9333-9340, https://doi.org/ 10.1021/acs.jafc.5b03572.

[12] B. Ozturk, S. Argin, M. Ozilgen, D.J. McClements, Formation and stabilization of nanoemulsion-based vitamin E delivery systems using natural surfactants: Quillaja saponin and lecithin, J. Food Eng. 142 (2014) 57-63, https://doi.org/10.1016/j. jfoodeng.2014.06.015.

[13] T.B. Schreiner, A. Santamaria-echart, A. Ribeiro, A.M. Peres, M.M. Dias, S.P. Pinho, M.F. Barreiro, Formulation and optimization of nanoemulsions using the natural surfactant saponin from Quillaja Bark, Molecules 25 (2020) 1-14, https://doi.org/ 10.3390/molecules25071538.

[14] Y. Yang, D.J. Mcclements, Vitamin E bioaccessibility: influence of carrier oil type on digestion and release of emulsified a-tocopherol acetate, Food Chem. 141 (2013) 473-481, https://doi.org/10.1016/j.foodchem.2013.03.033.

[15] M. Jarzebski, P. Siejak, W. Smulek, F. Fathordoobady, Y. Guo, J. Pawlicz, T. Trzeciak, P.L. Kowalczewski, D.D. Kitts, A. Singh, A.P. Singh, Plant extracts containing saponins affects the stability and biological activity of hempseed oil emulsion system, Molecules 25 (2020) 1-16, https://doi.org/10.3390/ molecules25112696.

[16] S. Böttcher, S. Drusch, Interfacial properties of saponin extracts and their impact on foam characteristics, Food Biophys. 11 (2016) 91-100, https://doi.org/10.1007/ s11483-015-9420-5.

[17] S. Mitra, S.R. Dungan, Micellar properties of Quillaja saponin. 1. Effects of temperature, salt, and $\mathrm{pH}$ on solution properties, J. Agric. Food Chem. 45 (1997) 1587-1595, https://doi.org/10.1021/jf960349z.

[18] T. Ralla, H. Salminen, J. Tuosto, J. Weiss, Formation and stability of emulsions stabilised by Yucca saponin extract, Int. J. Food Sci. Technol. 53 (2017) 1381-1388, https://doi.org/10.1111/ijfs.13715.

[19] T. Ralla, E. Herz, H. Salminen, M. Edelmann, C. Dawid, T. Hofmann, J. Weiss, Emulsifying properties of natural extracts from Panax ginseng L, Food Biophys. 12 (2017) 479-490, https://doi.org/10.1007/s11483-017-9504-5.

[20] T. Ralla, H. Salminen, M. Edelmann, C. Dawid, T. Hofmann, J. Weiss, Oat bran extract (Avena sativa L.) from food by-product streams as new natural emulsifier, Food Hydrocoll. 81 (2018) 253-262, https://doi.org/10.1016/j. foodhyd.2018.02.035.

[21] Z. Zhu, Y. Wen, J. Yi, Y. Cao, F. Liu, D.J. McClements, Comparison of natural and synthetic surfactants at forming and stabilizing nanoemulsions: Tea saponin, Quillaja saponin, and Tween 80, J. Colloid Interface Sci. 536 (2019) 80-87, https://doi.org/10.1016/j.jcis.2018.10.024.

[22] M. Bouhoute, N. Taarji, S. Vodo, I. Kobayashi, M. Zahar, H. Isoda, M. Nakajima, M. A. Neves, Formation and stability of emulsions using crude extracts as natural 
emulsifiers from Argan shells, Colloids Surf. A Physicochem. Eng. Asp. 591 (2020), 124536, https://doi.org/10.1016/j.colsurfa.2020.124536.

[23] A. Amin, M. Lotfy, M. Shafiullah, E. Adeghate, The protective effect of Tribulus terrestris in diabetes, Ann. N. Y. Acad. Sci. 1084 (2006) 391-401, https://doi.org/ 10.1196/annals.1372.005.

[24] M. Akram, H.M. Asif, N. Akhtar, P.A. Shah, M. Uzair, G. Shaheen, T. Shamim, S.M. A. Shah, K. Ahmad, Tribulus terrestris Linn.: a review article, J. Med. Plants Res. 5 (2011) 3601-3605, https://doi.org/10.5897/JMPR.9001271.

[25] P. Dixit, S. Ghaskadbi, H. Mohan, T.P.A. Devasagayam, Antioxidant properties of germinated fenugreek seeds, Phytother. Res. 19 (2005) 977-983, https://doi.org/ 10.1002/ptr.1769.

[26] F.O. of F.S. and A. Nutrition, Fenugreek, Extract (Trigonella foenum-graecum 1.), 2019.

[27] Y. Mimaki, M. Kuroda, A. Kameyama, A. Yokosuka, Y. Sashida, Aculeoside B, a new bisdesmosidic spirostanol saponin from the underground parts of Ruscus aculeatus, J. Nat. Prod. 61 (1998) 1279-1282, https://doi.org/10.1021/np9704563.

[28] D. Dinchev, B. Janda, L. Evstatieva, W. Oleszek, M.R. Aslani, I. Kostova, Distribution of steroidal saponins in Tribulus terrestris from different geographical regions, Phytochemistry 69 (2008) 176-186, https://doi.org/10.1016/j. phytochem.2007.07.003.

[29] A. Ivanova, I. Lazarova, P. Mechkarova, I. Semerdjieva, L. Evstatieva, Intraspecific variability of biologically active compounds of different populations of Tribulus terrestris in Thracian floristic region, Biotechnol. Biotechnol. Equip. 25 (2011) 2357-2361, https://doi.org/10.5504/BBEQ.2011.0041.

[30] S.P. Bhutani, S.S. Chibber, T.R. Seshadri, Flavonoids of the fruits and leaves of Tribulus terrestris: constitution of tribuloside, Phytochemistry 8 (1969) 299-303, https://doi.org/10.1016/S0031-9422(00)85828-8.

[31] N.A.M. Saleh, A.A. Ahmed, M.F. Abdalla, Flavonoid glycosides of Tribulus pentandrus and T. Terrestris, Phytochemistry 21 (1982) 1995-2000, https://doi. org/10.1016/0031-9422(82)83030-6.

[32] T.S. Wu, L.S. Shi, S.C. Kuo, Alkaloids and other constituents from Tribulus terrestris, Phytochemistry 50 (1999) 1411-1415, https://doi.org/10.1016/S0031 9422(97)01086-8.

[33] C.A. Bourke, G.R. Stevens, M.J. Carrigan, Locomotor effects in sheep of alkaloids identified in Australian Tribulus terrestris, Aust. Vet. J. 69 (1992) 163-165, https://doi.org/10.1111/j.1751-0813.1992.tb07502.x.

[34] G. Petkov, Enhancement of Tribulus terrestris L. yield by supplement of green house seedlings, Biotechnol. Biotechnol. Equip. 25 (2011) 2366-2368, https://doi. org/10.5504/BBEQ.2011.0039.

[35] A.L. Lv, N. Zhang, M.G. Sun, Y.F. Huang, Y. Sun, H.Y. Ma, H.M. Hua, Y.H. Pei, One new cinnamic imide dervative from the fruits of Tribulus terrestris, Nat. Prod. Res. 22 (2008) 1007-1010, https://doi.org/10.1080/14786410701654867.

[36] Y.H. Song, D.W. Kim, M.J. Curtis-Long, C. Park, M. Son, J.Y. Kim, H.J. Yuk, K. W. Lee, K.H. Park, Cinnamic acid amides from Tribulus terrestris displaying uncompetitive $\alpha$-glucosidase inhibition, Eur. J. Med. Chem. 114 (2016) 201-208, https://doi.org/10.1016/j.ejmech.2016.02.044.

[37] S.A. Wani, P. Kumar, Fenugreek: a review on its nutraceutical properties and utilization in various food products, J. Saudi Soc. Agric. Sci. 17 (2018) 97-106, https://doi.org/10.1016/j.jssas.2016.01.007.

[38] S.C. Jain, A. Madhu, Regulation of trigonellin in Trigonella species by chemical mutagenic treatments, Indian Drugs 26 (1988) 14-16.

[39] C.P. Khare. Indian Herbal Remedies, 1st ed., Springer-Verlag, Berlin Heidelberg, Berlin, 2004.

[40] G.A. Petropoulos. Fenugreek, 1st ed., Taylor \& Francis, New York, 2002.

[41] M.M. Naidu, B.N. Shyamala, J.P. Naik, G. Sulochanamma, P. Srinivas, Chemical composition and antioxidant activity of the husk and endosperm of fenugreek seeds, LWT - Food Sci. Technol. 44 (2011) 451-456, https://doi.org/10.1016/j. lwt.2010.08.013.

[42] E. De Combarieu, M. Falzoni, N. Fuzzati, F. Gattesco, A. Giori, M. Lovati, R. Pace, Identification of Ruscus steroidal saponins by HPLC-MS analysis, Fitoterapia 73 (2002) 583-596, https://doi.org/10.1016/s0367-326x(02)00220-4.

[43] C. Dunouau, R. Belle, A. Oulad-al, Triterpenes and sterols from Ruscus aculeatus, Planta Med. 62 (1996) 190-191, https://doi.org/10.1055/s-2006-957858.

[44] N. Hadzifejzovic, J. Kukic-Markovic, S. Petrovic, M. Sokovi, J. Glamoclika, D. Stojkovic, A. Nahrstedt, Bioactivity of the extracts and compounds of Ruscus aculeatus L. and Ruscus hypoglossum L, Ind. Crop. Prod. 49 (2013) 407-411, https://doi.org/10.1016/j.indcrop.2013.05.036.

[45] E.M. Agency, Assessment Report on Ruscus Aculeatus L. Rhizoma, 2008.

[46] A. Berthod, M. Hassoun, M.J. Ruiz-Angel, Alkane effect in the Arizona liquid systems used in countercurrent chromatography, Anal. Bioanal. Chem. 383 (2005) 327-340, https://doi.org/10.1007/s00216-005-0016-7.

[47] O. Ferreira, S.P. Pinho, Solubility of flavonoids in pure solvents, Ind. Eng. Chem. Res. 51 (2012) 6586-6590, https://doi.org/10.1021/ie300211e.

[48] S.M. Vilas-Boas, V. Vieira, P. Brandão, R.S. Alves, J.A.P. Coutinho, S.P. Pinho, O. Ferreira, Solvent and temperature effects on the solubility of syringic, vanillic or veratric acids: experimental, modeling and solid phase studies, J. Mol. Liq. 289 (2019), 111089, https://doi.org/10.1016/j.molliq.2019.111089.

[49] T.C. Obasi, R. Moldovan, A. Toiu, C. Braicu, E. Bodoki, I. Berindan, I. Oniga, R. Sandulescu, R. Oprean, Molecular-trapping in emulsion's monolayer: a new strategy for production and purification of bioactive saponins, Sci. Rep. 7 (2017) 1-9, https://doi.org/10.1038/s41598-017-15067-4.

[50] A. Cano-Medina, H. Jiménez-Islas, L. Dendooven, R.P. Herrera, G. GonzálezAlatorre, E.M. Escamilla-Silva, Emulsifying and foaming capacity and emulsion and foam stability of sesame protein concentrates, Food Res. Int. 44 (2011) 684-692, https://doi.org/10.1016/j.foodres.2010.12.015.
[51] A.L. Lupatini Menegotto, L.E.S. de Souza, L.M. Colla, J.A.V. Costa, E. Sehn, P.R. S. Bittencourt, É.L. de Moraes Flores, C. Canan, E. Colla, Investigation of technofunctional and physicochemical properties of Spirulina platensis protein concentrate for food enrichment, LWT 114 (2019), 108267, https://doi.org/ 10.1016/j.lwt.2019.108267.

[52] S.M. Dizaj, Preparation and study of vitamin A palmitate microemulsion drug delivery system and investigation of co-surfactant effect, J. Nanostruct. Chem. 5 (2013) 1-6, https://doi.org/10.1186/2193-8865-3-59.

[53] S. Mitra, S.R. Dungan, Cholesterol solubilization in aqueous micellar solutions of Quillaja saponin, bile salts, or nonionic surfactants, J. Agric. Food Chem. 49 (2001) 384-394, https://doi.org/10.1021/jf000568r.

[54] P.S. Piispanen, M. Persson, P. Claesson, T. Norin, Surface properties of surfactants derived from natural products. Part 1: syntheses and structure/property relationships - solubility and emulsification, J. Surfactants Deterg. 7 (2004) 147-159, https://doi.org/10.1007/s11743-004-0298-6.

[55] W.D. Bancroft, The theory of emulsification V, J. Phys. Chem. 17 (1913) 501-519.

[56] C.L. Reichert, H. Salminen, J. Weiss, Quillaja saponin characteristics and functional properties, Annu. Rev. Food Sci. Technol. 10 (2019) 43-73, https://doi.org/ 10.1146/annurev-food-032818-122010.

[57] S. Balakrishnan, S. Varughese, A.P. Deshpande, Micellar characterisation of saponin from Sapindus mukorossi, Tenside Surfactants Deterg. 43 (2006) 262-268, https://doi.org/10.3139/113.100315.

[58] M. Wink, Medicinal plants: a source of anti-parasitic secondary metabolites, Molecules 17 (2012) 12771-12791, https://doi.org/10.3390/ molecules171112771.

[59] M.S. Almutairi, M. Ali, Direct detection of saponins in crude extracts of soapnuts by FTIR, Nat. Prod. Res. 29 (2015) 1271-1275, https://doi.org/10.1080/ 14786419.2014.992345.

[60] U. Aswar, S.L. Bodhankar, V. Mohan, P.A. Thakurdesai, Effect of furostanol glycosides from Trigonella foenum-graecum on the reproductive system of male albino rats, Phytother. Res. 24 (2010) 1482-1488, https://doi.org/10.1002/ptr.3129.

[61] C. Zhang, S. Khoo, P. Swedlund, Y. Ogawa, Y. Shan, S.Y. Quek, Fabrication of spray-dried microcapsules containing noni juice using blends of maltodextrin and gum acacia: physicochemical properties of powders and bioaccessibility of bioactives during in vitro digestion, Foods 9 (2020) 1-17, https://doi.org/ $10.3390 /$ foods 9091316.

[62] W.E. Walkowicz, A. Fernández-Tejada, C. George, F. Corzana, J. Jiménez-Barbero, G. Ragupathi, D.S. Tan, D.Y. Gin, Quillaja saponin variants with central glycosidic linkage modifications exhibit distinct conformations and adjuvant activities, Chem. Sci. 7 (2016) 2371-2380, https://doi.org/10.1039/c5sc02978c.

[63] S. Mitra, S.R. Dungan, Micellar properties of quillaja saponin. 2. Effect of solubilized cholesterol on solution properties, Colloids Surf. B Biointerfaces 17 (2000) 117-133, https://doi.org/10.1016/S0927-7765(99)00088-0.

[64] K. Golemanov, S. Tcholakova, N. Denkov, E. Pelan, S.D. Stoyanov, The role of the hydrophobic phase in the unique rheological properties of saponin adsorption layers, Soft Matter 10 (2014) 7034-7044, https://doi.org/10.1039/c4sm00406j.

[65] K.G.O. Bezerra, I.J.B. Durvala, I.A. Silva, F.C.G. Almeida, Y.T.F. Melo, R.D. Rufino, L.A. Sarubbo, Emulsifying capacity of biosurfactants from Chenopodium quinoa and pseudomonas aeruginosa UCP 0992 with focus of application in the cosmetic industry, Chem. Eng. Trans. 79 (2020) 211-216, https://doi.org/10.3303/ CET2079036.

[66] K. Abdolmaleki, M. Amin, R. Mohammadi, The effect of $\mathrm{pH}$ and salt on the stability and physicochemical properties of oil-in-water emulsions prepared with gum tragacanth, Carbohydr. Polym. 140 (2016) 342-348, https://doi.org/10.1016/j. carbpol.2015.12.081.

[67] Y. Shishikura, S. Khokhar, B.S. Murray, Effects of tea polyphenols on emulsification of olive oil in a small intestine model system, J. Agric. Food Chem. 54 (2006) 1906-1913, https://doi.org/10.1021/jf051988p.

[68] J.T. de Faria, E.B. de Oliveira, V.P.R. Minim, L.A. Minim, Emulsifying properties of $\beta$-lactoglobulin and Quillaja bark saponin mixtures: effects of number of homogenization passes, $\mathrm{pH}$, and $\mathrm{NaCl}$ concentration, Int. J. Food Prop. 20 (2017) 1643-1654, https://doi.org/10.1080/10942912.2016.1217007.

[69] V. Ulaganathan, L. Del Castillo, J.L. Webber, T.T.M. Ho, J.K. Ferri, M. Krasowska, D.A. Beattie, The influence of $\mathrm{pH}$ on the interfacial behaviour of Quillaja bark saponin at the air-solution interface, Colloids Surf. B Biointerfaces 176 (2019) 412-419, https://doi.org/10.1016/j.colsurfb.2019.01.017.

[70] W. Oleszek, A. Hamed, Saponin-based surfactants, in: M. Kjellin, I. Johansson (Eds.), Surfactants from Renewable Resources, 1st ed., John Wiley \& Sons Ltd, 2010.

[71] X. Jiang, B.W. Strobel, N. Cedergreen, Y. Cao, H.C.B. Hansen, Stability of saponin biopesticides: hydrolysis in aqueous solutions and lake waters, Environ. Sci. Process. Impacts 21 (2019) 1204-1214, https://doi.org/10.1039/c9em00012g.

[72] P.S. Piispanen, M. Persson, P. Claesson, T. Norin, Surface properties of surfactants derived from natural products. Part 2: structure/property relationships - foaming, dispersion, and wetting, J. Surfactants Deterg. 7 (2004) 161-167, https://doi.org/ 10.1007/s11743-004-0299-5.

[73] P. Gane, J. Schoelkopf, Procedure for the Grinding of Mineral Materials and Binders in an Aqueous Medium Implementing a Reverse Emulsion of an Acrylamide Polymer with an Acrylic Monomer, EP 1990376 B1, 2011.

[74] C.L. Reichert, H. Salminen, G. Badolato Bönisch, C. Schäfer, J. Weiss, Concentration effect of Quillaja saponin - co-surfactant mixtures on emulsifying properties, J. Colloid Interface Sci. 519 (2018) 71-80. 〈https://www.sciencedirect. com/science/article/abs/pii/S0021979718301231>.

[75] N. Taarji, M. Bouhoute, H. Melanie, A. Hafidi, I. Kobayashi, M. Neves, K. Tominaga, H. Isoda, M. Nakajima, Interfacial and emulsifying properties of purified glycyrrhizin and non-purified glycyrrhizin-rich extracts from liquorice 
root (Glycyrrhiza glabra), Food Chem. 337 (2021), 127949, https://doi.org/ 10.1016/j.colsurfa.2020.126006.

[76] P.A. Winsor, Hydrotropy, solubilisation and related emulsification processes. Part I, Trans. Faraday Soc. 44 (1947) 376-398, https://doi.org/10.1039/ TF9484400376.

[77] M. de Villiers, Surfactants and emulsifying agents, in: J.E. Thompson (Ed.), A Practical Guide to Contemporary Pharmacy Practice, 3rd ed., Lippincott Williams \& Wilkins, 2009, pp. 251-256.
[78] C.L. Reichert, H. Salminen, B.H. Leuenberger, J. Hinrichs, J. Weiss, Miscibility of Quillaja saponins with other co-surfactants under different $\mathrm{pH}$ values, J. Food Sci. 80 (2015) E2495-E2503, https://doi.org/10.1111/1750-3841.13097.

[79] A. Froelich, T. Osmałek, A. Snela, P. Kunstman, B. Jadach, M. Olejniczak, G. Roszak, W. Białas, Novel microemulsion-based gels for topical delivery of indomethacin: formulation, physicochemical properties and in vitro drug release studies, J. Colloid Interface Sci. 507 (2017) 323-336. 